\title{
Der schöne Schein
}

\section{Banknoten als Untersuchungsgegenstand einer visuellen Soziologie}

\section{Von Christian Thiel}

Zusammenfassung: Der Aufsatz befasst sich mit der Visualität von Banknoten. Mittels einer aus der Kunstgeschichte abgeleiteten Heuristik wird der bisherige (spärliche) Forschungsstand systematisch aufgearbeitet. Dargestellt werden die historisch sich wandelnden Motive, der Einfluss des Produktionskontextes sowie die durch die Banknoten vermittelten Botschaften (intendierte Rezeption). Die daraus abgeleitete These ist, dass die Gestaltung von Banknoten prinzipiell inhaltsoffen ist, solange sie in einer geldtypischen Bildsprache erfolgt.

\section{Einleitung}

„Es wäre eine beschreibende Analysis der Banknoten zu liefern. Ein Buch, dessen grenzenlose Kraft der Satire ihresgleichen nur in der Kraft seiner Sachlichkeit hätte. Denn nirgends mehr als in diesen Dokumenten gebärdet der Kapitalismus sich naiv in seinem heiligen Ernst. Was hier an unschuldigen Kleinen um Ziffern spielt, als Göttinnen Gesetzestafeln hält und an gereiften Helden vor Münzeinheiten sein Schwert in die Scheide steckt, das ist eine Welt für sich: Fassadenarchitektur der Hölle. “ (Benjamin 1928: 72)

Visualität zählt zu den relevantesten Phänomenen der Gegenwart. Alle Bereiche des Alltagslebens werden von einer wahren ,Bilderflut ‘ überschwemmt, deren Ausmaß nicht zuletzt durch die Neuen Medien stetig zunimmt. So ist es nur konsequent, dass sich in den letzten 10 bis 15 Jahren zahlreiche wissenschaftliche Disziplinen, so auch die Soziologie, den Bildern, Filmen, Videos und anderen Formen visueller Kommunikation zuwenden - eine Entwicklung, die unter dem Schlagwort ,Iconic Turn' subsummiert werden kann. Überraschend erscheint allerdings, dass gerade eines der am häufigsten verbreitetsten visuellen Produkte der Moderne kaum Gegenstand der Forschung ist: die Banknote.

Der vorliegende Beitrag ${ }^{1}$ versucht aus der Perspektive einer visuellen Soziologie eine Analyse von Banknoten. Grundsätzlich geht es dabei um die Frage, was auf dem Papiergeld dargestellt ist und wie sich dies soziologisch deuten lässt. Angesichts der Vielfältigkeit dieses seit einigen 100 Jahren weltweit existierenden Untersuchungsgegenstandes erscheint es sinnvoll, dies zunächst nicht in einer (zwangsläufig begrenzten) Einzelstudie anzugehen, sondern mittels einer systematischen Durchsicht des bisherigen Forschungsstandes. So kann gleichzeitig der Forschungsgegenstand ,Banknote' für künftige empirische Untersuchungen im Rahmen der visuellen Soziologie erschlossen werden. Dazu wird erstens dargestellt, warum Banknoten überhaupt ein lohnender Untersuchungsgegenstand sind und welche Forschungen es bislang dazu gibt. Nach einer zweitens knappen Darstellung der historischen Entwicklung und momentanen Verbreitung des Papiergeldes erfolgen drittens grundlegende funktionale, zeichentheoretische und ikonographische Überlegungen. Entsprechend dieser werden viertens mit Hilfe der bisherigen Arbeiten die die konkrete Visualität bestimmenden Aspekte der Bildgestaltung, des Produktionsprozesses und der (intendierten bzw. tatsächlichen) Rezeption diskutiert. Der abschließende Ausblick entwickelt fünftens aus der Vielfalt der dargestellten Ergebnisse einige dezidiert soziologische Perspektiven und gibt einen kurzen methodischen Ausblick.

1 Für wertvolle Hinweise danke ich einem anonymen Gutachter.

Soziale Welt 64 (2013), S. $191-216$ 


\section{Banknoten im Blickpunkt der Forschung}

Die Beschränkung auf Banknoten erscheint zunächst vielleicht seltsam - schließlich sind Banknoten ebenso wie Münzen oder Giralgeld in letzter Konsequenz schlichtweg, Geld‘. Geld ist jedoch immer an eine bestimmte Gegenständlichkeit gebunden (und seien das die Bits und Bytes auf Festplatten). Und ein Aspekt dieser Materialität ist die Ästhetik, denn ,die quantitative Größe Geld [hat sich] in ihrer empirischen Gestalt doch stets der ästhetischen Qualitäten zumindest als eines rhetorischen Mittels bedient“" (Gabriel 2002: $27 \mathrm{f}$ ). Man könnte nun diverse Geldformen entsprechend analysieren: Münzen, Banknoten, Gutscheine, selbst die verschiedenen Darstellungsweisen von Giralgeld. Gegenstand der folgenden Betrachtung sind jedoch ausschließlich (offizielle) Banknoten. Diese Auswahl ist natürlich willkürlich. Wichtig für die Analyse der konkreten Materialität unterschiedlicher Geldformen ist nur, dass man eine Wahl trifft und nicht pauschal ,Geld' untersucht. Natürlich kann auf Banknoten ein wesentlich komplexeres ikonographisches Programm realisiert werden als auf Münzen; auch ist die Diskrepanz zwischen Material- und Nominalwert - und damit der Legitimierungsdruck - wesentlich größer als bei Metallgeld. Dennoch lohnt jede Geldform für sich genommen eine Untersuchung. Bislang haben nur wenige Studien Geld in seiner konkreten Materialisation als Geldschein ernst genommen und versucht, diese „Kunst in Milliardenauflage“ (Gabriel 2002: 15) analytisch aufzuschlüsseln. Dabei sind Banknoten aus mehreren Gründen ein äußerst lohnender Untersuchungsgegenstand: Erstens aufgrund ihrer Universalität. Papiergeld gibt es nicht nur weltweit, ${ }^{2}$ sondern es ist auch ein stets präsenter Bestandteil des alltäglichen Lebens. Zweitens aufgrund der Selektivität des Designs. Der durch die Stückelung und das Format begrenzte Platz erfordert klare Entscheidungen, was abgebildet wird und was nicht. Drittens aufgrund der regelmäßigen Neugestaltung, die aus Gründen von Fälschungssicherheit und Inflation im Schnitt alle zehn bis 20 Jahre erfolgt und interessante Zeitvergleiche (über hunderte von Jahren) möglich macht (Hymans 2004: 7; Hymans 2005: 317).

Angesichts dessen ist es verwunderlich, dass sich die Forschung - weder Geschichtswissenschaft oder Kunstgeschichte noch Soziologie oder Politologie, ja nicht einmal die Numismatik - kaum explizit mit den ästhetischen bzw. ikonographisch-ikonologischen Aspekten von Banknoten auseinandergesetzt hat. Die wenigen Studien, die es dazu gibt, verteilen sich wie folgt: Neben allgemeinen Nachschlagewerken für Sammler (etwa Pick 1992 oder der in drei Bänden erscheinende ,Standard Catalog of World Paper Money“) gibt es etliche Publikationen aus dem Bereich der Währungsgeschichte, die die Entstehung und Verbreitung des Papiergeldes als einen Schritt der monetären Entwicklung nachzeichnen (etwa Klose 1999; Sedillot 1992; Weimer 1994; explizit zu Papiergeld siehe: Angus 1975; Brion / Moreau 2001; Deutsche Bundesbank 1970; Heij 2012; Nathorst-Böös 1970). Der Großteil der einschlägigen Publikationen, meist aus Politologie, Geschichte oder politische Geographie, befasst sich mit dem Zusammenhang von Papiergeld und nationaler Identität bzw. Nationenbildung (Blaazer 2002; Fuller 2008; Hymans 2004; Penrose 2011; Pointon 1998; Veselkova / Horvath 2011; Wallach 201). Die Schwerpunkte sind jeweils leicht unterschiedlich: Manche konzentrieren sich auf bestimmte Zeitperioden (etwa die Entstehung der Nationalstaaten im 19. Jahrhundert; siehe Gilbert / Helleiner 1999 a; Helleiner 2003), andere fragen nach konkreten Wirkungen (etwa räumlich-symbolische Abgrenzungen durch das Medium Geld; siehe Gilbert 1998; Raento et al. 2004; Unwin / Hewitt 2001). Mal wird der Fokus auf eine spezielle Währung (bspw. den Euro, siehe Hymans 2006; Kaelberer 2004, 2005; Risse 2003; Winter 2007) oder auf eine bestimmte Region bzw. Kulturraum gelegt (Hymans 2005; Mwangi 2002). Diese politologi-

2 Fast jedes Land der Welt hat eigene Banknoten. Ausnahmen sind Währungsunionen: Der Euro gilt in 23 europäischen Staaten, der East Caribbean Dollar in sechs karibischen Staaten und zwei britischen Überseegebieten, der CFA-Franc BCEAO in acht westafrikanischen Staaten und der CFA-Franc BEAC in sechs zentralafrikanischen Staaten. 
sche Perspektive vermischt sich stark mit der Geschichtsforschung, auch wenn hier teils eigene (unpolitische) Akzente gesetzt werden, beispielsweise in technikgeschichtlicher (Robertson 2005; Uemuro 2007) oder numismatischer Hinsicht (Hewitt / Keyworth 1987; Hewitt 1994, 1995; Hymans 2005; Klüßendorf 2007; Tschoegl 2002;). Für die Ökonomie, konkret für das Bankwesen, sind Banknoten ein Teil ihres Aufgabengebietes. Dementsprechend gibt es eine Vielzahl von Publikationen (v.a. von nationalen Zentralbanken oder der europäischen Zentralbank), die allerdings zu weiten Teilen sehr deskriptiv-statistisch (Bargeldmenge, -verwendung, -zirkulation etc.) bzw. praktisch ausgerichtet sind (Sicherheitsmerkmale, Banknotenkreislauf \& -recycling etc.). Nur selten befasst man sich mit der konkreten Gestaltung bzw. deren Wirkung (etwa Heij 2009, 2012). Aus anderen Disziplinen kommen nur sehr sporadisch Beiträge, so aus der Ethnologie (Lambek 2001; Tappe 2007), der Kunstgeschichte (Athanassakis 2008; Gabriel 2002, 2009, Schramm 1985), der Literaturwissenschaft (Hörisch 1996), der Soziologie (Miklautz 2005; Priddat 2003) oder der Kulturwissenschaften (Cox / SchmidtHannisa 2007; Löffler 2010).

Zusammenfassend betrachtet lässt sich der Forschungsstand nur als ein unverbundener Flickenteppich beschreiben. Die bisherigen Publikation beinhalten - und das ist kaum überraschend - unterschiedliche disziplinäre Herangehensweisen, wählen unterschiedliche Blickwinkel (Fokus auf bestimmte Zeiten, Länder, Gebiete, Banknotenproduzenten usw.) oder (falls sie empirisch arbeiten) unterschiedliche methodische Herangehensweisen. Zudem handelt es sich in weiten Teilen um unverbunden stehende Einzelstudien. Um diese nun für eine visuelle Soziologie nutzbar zu machen, gilt es sie systematisch zusammenzutragen und zu interpretieren. Notwendiges Hintergrundwissen hierfür (im Sinne eines Korrektivprinzips der Interpretation) sind jedoch Kenntnisse der Entstehungsgeschichte des Papiergeldes.

\section{Das Papiergeld früher und heute}

Das Papiergeld entstand erst relativ spät in der Geschichte des Geldes. Die allerersten Geldformen waren Natural- und Warengelder (Muscheln, Salz, Kakaobohnen, Gewürze, Zähne, Felle etc.), die im Laufe der Zeit von Edelmetallen abgelöst wurden. Erste Vorformen des Papiergeldes entstanden ab dem 13. Jahrhundert in Europa. ${ }^{3}$ Damals stellten die Vorläufer der modernen Banken in den italienischen Handelsstädten Wechsel und Schuldscheine aus (Weatherford 1999: 100ff). Hinzu kamen Quittungen für eingezahlte Geldeinlagen, die meist verzinst wurden. Immer häufiger wurden diese zunächst nicht übertragbaren Inhaberscheine indossiert und statt Bargeld zur Zahlung verwendet - so geschehen etwa bei der 1609 in Amsterdam gegründeten Wisselbank (Bender 1004: 14) oder bei den von englischen Goldschmieden ausgestellten ,goldsmith notes' (North 1994: 13). Die ersten ,offiziellen' Banknoten ${ }^{4}$ emittierte die Bank of Stockholm 1661; dies allerdings nur knappe zwei Jahre lang. Denn schon damals zeigten sich grundlegenden Probleme des Papiergeldes: Fälschung und Inflation in Folge von verantwortungsloser Überemission. Trotzdem verbreitete sich das Papiergeld rasch

3 In China wurde das Papiergeld bereits um 960 erfunden (Sedillot 1992: 164). Schon damals zeigten sich einige Grundprobleme des Papiergeldes: Es wurde häufig gefälscht und unterlag immer wieder starken Inflationen (weil die Kaiser häufig gewaltige Mengen Geld drucken ließen).

4 ,Offizielle' bzw. ,richtige' Banknoten aus mehreren Gründen: Erstens, weil hier auf Zinszahlungen seitens der Bank verzichtet wurde, es sich also um ein Zahlungsmittel und nicht um eine Geldanlage handelte. Zweitens weil die Bank of Stockholm zwar rechtlich eine private Firma, de facto aber staatlich war (wurde durch Finanzministerium überwacht, durfte Zollbescheinigungen ausstellen, musste Hälfte von Gewinn an den Staat abführen). Drittens, weil die Bank mehr Banknoten emittierte, als an Edelmetalleinlagen hinterlegt war - das Geld also zumindest teilweise durch ein staatliches Wertversprechen gedeckt war (Brion / Moreau 2001: 3 f). Obwohl dies etwas genuin Neues darstellte, wurden die Banknoten recht gerne angenommen. Dies mag auch daran liegen, dass sie die damals als Zahlungsmittel dienenden platmynt (Plattenmünzen) ersetzten, die mit einer Größe von jeweils 33 x $68 \mathrm{~cm}$ und einem Gewicht von knapp $20 \mathrm{~kg}$ doch recht sperrig waren. 
- wahrscheinlich, weil es dem aufblühenden Kapitalismus jenes zur Verfügung stellte, was er unbedingt brauchte: Geld für Zahlungsverkehr und Investitionen. Damals war schlichtweg zu wenig Edelmetall vorhanden, um den vorhandenen Bedarf zu stillen. Zudem war Papiergeld praktischer, einfacher zu transportieren und damit sicherer als die oft sperrigen Münzen. Im 18. Jahrhundert entstanden deswegen allerorts notenausgebende private Banken, die mal mehr, mal (sehr viel) weniger vom Staat kontrolliert wurden (Brion / Moreau 2001: 8). Unter anderem aufgrund der zahlreichen Krisen in Folge von Überemission und Fälschungen im Laufe des 19. Jahrhunderts wurden diese zunehmend verstaatlicht. Die neu entstandenen Nationalstaaten wollten Kontrolle über das ,öffentliche Gut' Geld. Zudem machte der sich verstärkende wirtschaftliche Austausch eine Vereinheitlichung notwendig. Dies beendete die damals bunte Währungsvielfalt, bei der eine Vielzahl an Münzen aus verschiedenen Zeiten und von unterschiedlichen Herrschern teils aufwändig mittels schwankender Wechselkurse ineinander umgerechnet werden mussten (Gilbert / Helleiner 1999 b: 3). ${ }^{5}$ Aufgrund der verstärkten Rolle des Staates im wirtschaftlichen Leben (etwa durch Verbreitung von Postämter, von staatlichen Banken, von Eisenbahnen, der Einführung von allgemeiner Wehrpflicht und eines allgemeinen Steuersystems) konnten diese Geldreformen erfolgreich durchgesetzt werden (Knapp 1924). Damit wandelte sich auch die rechtliche Stellung des Geldes. Als das ursprünglich privatrechtlich abgesicherte Schuldverhältnis, das eine Banknote symbolisiert, zunehmend für Steuerzahlungen eingesetzt werden konnte, erhielt es eine Art ,offizieller Garantie'. Es wurde zum ,gesetzlichen Zahlungsmittel' erklärt und damit zu einem Gegenstand des öffentlichen Rechts (Brion / Moreau 2001: 22). Neben der Verstaatlichung kam es im 19. Jahrhundert auch zu einer ,Demokratisierung' des Papiergeldes. Lange Zeit waren Banknoten den Reichen vorbehalten, denn schon aus Furcht vor Fälschungen wurden hauptsächlich hohe Nennwerte gedruckt. ${ }^{6}$ Paradoxerweise führten nun gerade die vielen Kriege und Revolutionen, Währungskrisen und Bank-Runs sowie der damit verbundene Vertrauensverlust des Papiergeldes zu seiner weiten Verbreitung. Denn jedes Mal wenn das Papiergeld an Glaubwürdigkeit verlor, begannen die Menschen Edelmetalle zu horten; dieses benötigten allerdings die Banken für die (zumindest partielle) Deckung ihrer Banknoten (Brion / Moreau 2001: 19). Um das Geschäft am Laufen zu halten, wurden deswegen immer kleinere Denominationen herausgegeben, wodurch immer größere Bevölkerungsanteile Zugang zu Papiergeld erhielten (Gilbert / Helleiner 1999b: 5). So bildete sich im frühen 20. Jahrhundert das Modell eines einheitlichen, universell verbreiteten, gesetzlich anerkannten Zahlungsmittels heraus, das ausschließlich von einer nationalen Zentralbank herausgegeben werden darf. England setzte dies als eine der ersten Nationen um, die USA und viele europäische Länder folgten. Nach dem Ersten Weltkrieg adaptierten weite Teile Asiens und Lateinamerikas dieses Modell; nach dem Zweiten Weltkrieg verbreitete es sich auch in Afrika (Gilbert / Helleiner 1999 b: 4). Seither waren vor allem zwei weitere Entwicklungen maßgeblich: Erstens die Aufhebung des Goldstandards 1971 durch Nixon. Bis

5 Dabei wurden nicht nur unterschiedliche Währungen durch eine Nationalwährung ersetzt, sondern diese auch am metrischen System ausgerichtet. Die USA stellten 1782 ihre Währung als erste auf das Dezimalsystem um, die Engländer als letzte 1971. Bei letzteren herrschte davor eine verwirrende Mischung: So entsprach etwa ein Shilling 12 Pence; jeder Pence zerfiel in vier Farthings. Ein Pfund bestand aus 20 Shilling und die Guinee, eine größere Goldmünze, betrug ein Pfund plus ein Shilling (Weatherford 1999: 181).

6 Beispielsweise war es der Bank of England gemäß Satzung bis 1793 verboten, Banknoten von weniger als 20 Pfund (ca. ein Monatsgehalt eines Seemannes) herauszugeben. Die Banque de France emittierte erst 1847 eine Banknote von weniger als 500 Franc (etwa ein Jahreslohn eines Arbeiters) (Bender 2004: $18)$. 
dahin war das Papiergeld zumindest partiell mit Edelmetall gedeckt. ${ }^{7}$ Seither existieren nur noch Fiat-Währungen, die ausschließlich auf institutionalisiertem Vertrauen beruhen. Zweitens der Siegeszug des Giralgeldes. Schon im 19. Jahrhundert bauten Aktien- und Privatbanken das Geschäft der kurzfristigen Kreditgewährung mittels Wechsel, Scheck und Kontokorrentkredit aus. Schon damals machte der Buchgeldverkehr mehr als die Hälfte des Zahlungsverkehrs aus. Heutzutage stellen die Sichtguthaben bei Banken bereits 85\% der Zahlungsmittel (Geldmenge M1) dar (Huber 2004: 5).

Angesichts dessen werden immer wieder Nachrufe auf das Papiergeld verfasst. Dies erscheint allerdings etwas verfrüht, wie einige Zahlen verdeutlichen mögen: Ende 2010 waren Eurobanknoten im Wert von 840 Milliarden $€$ im Umlauf(EZB 2011: 85). Verwendet werden diese ungefähr zu einem Drittel als Transaktionskasse, ein weiteres Drittel entfällt auf die Kassenbestände der Banken, der Rest dient als Wertaufbewahrungsmittel (EZB 2011: 85). Schätzungsweise wurden allein 2008 zwischen 1.800 bis 2.100 Milliarden $€$ an Geldausgabeautomaten und Bankschaltern abgehoben. Im Vergleich dazu belaufen sich die elektronischen Zahlungen mit Debit- und Kreditkarten im selben Jahr auf knapp 1.000 Milliarden $€$ (EZB 2011: 87). Was die Bargeldverwendung betrifft, wird der Umfrage zufolge ein sehr großer Anteil (87\%) der kleinen Einkäufe (von weniger als $20 €$ ) bar bezahlt. Auch Käufe bis $100 €$ werden mehrheitlich (55\%) bar abgewickelt. Ein nicht unerheblicher Anteil (20\%) zahlt sogar Beträge bis zu $1000 €$ in bar. Selbst Großbetragszahlungen von über $10.000 €$ werden zu 4\% noch mit Banknoten abgewickelt (EZB 2011: 89). ${ }^{8}$ Angesichts dessen stellt die Europäische Zentralbank klar: „Mit Blick auf die Zukunft ist mittelfristig davon auszugehen, dass Bargeld ein wichtiges Zahlungsmittel bleiben und der Einfluss neuer Zahlungsinstrumente nur moderat sein wird.“ (EZB 2011: 95)

\section{Perspektiven und Fragestellungen}

Bevor man die Visualität von Banknoten analysieren kann, gilt es sich klar zu machen, was ihre Besonderheiten sind und welche epistemologischen und methodologischen Konsequenzen sich daraus ergeben. Banknoten sind schließlich keine beliebigen drucktechnischen Erzeugnisse oder simple Kunstwerke - sie sind in erster Linie Geld. Ausgegangen wird dazu von einer dezidiert soziologischen Perspektive, die, wo erforderlich, an der einen oder anderen Stelle ergänzt wird.

Für die Soziologie ist Geld ein Informationsträger (Mittermeier 2006), es vermittelt Tauschberechtigungs- wie auch Wertinformationen und ermöglicht dadurch Handlungskoordinierung. Geld wird dementsprechend häufig als ein ,symbolisch generalisiertes Kommunikationsmedium“ (Luhmann 1987: 222) konzipiert, das sich ,aus der normalen Sprache als ein auf Standardsituationen (des Tausches) zugeschnittener Spezialcode abgezweigt" (Habermas 1985: 406) hat. Bei diesem ,Spezialcode" geht es nur noch darum, ob Geldflüsse in Gang gesetzt werden oder ausbleiben, also letztlich um Zahlen oder Nicht-Zahlen (Luhmann 1998: 92ff). Diese Vereinfachung hilft das Referenzproblem des Wirtschaftssystems, die Knappheit der Güter, zu regeln, indem es die damit verbundene Komplexität reduziert und die Wahr-

7 Während des 19. und 20. Jahrhunderts gab es heftige Debatten, ob eine Währung mit Gold oder mit Gold und zusätzlich Silber (Bimetallismus) gedeckt sein soll (Vilar 1984: 324ff; Weimer 1994: 119). Das Gold setzte sich durch, spätestens mit der Konferenz von Bretton Wood, in der ein internationales Währungssystem von festen Wechselkursen mit dem goldhinterlegten Dollar als Leitwährung vereinbart wurde.

8 Allerdings zeigen sich im Ländervergleich deutliche Unterschiede. So wird Bargeld in Österreich, Italien und Spanien häufiger und zudem für größere Transaktionen eingesetzt; in Frankreich und den Niederlanden ist die Bargeldverwendung am geringsten (EZB 2011: 95). 
scheinlichkeit von (wirtschaftlicher) Kommunikation erhöht (Luhmann 1987: 222). ${ }^{9}$ Diesen Kommunikationsprozess materialisieren die Banknoten. Offen bleibt jedoch, welche Rolle der konkreten Materialität - und damit auch der Visualität - von Banknoten zukommt. Dies wird bisher in den soziologischen Geldtheorien nicht thematisiert. Im Gegenteil. Geld gilt als Gegenstand von „höchster Künstlichkeit“ und „Unanschaulichkeit“, der „Substanz repräsentiert, ohne sie sein oder auch nur abbilden zu müssen“ (Blumenberg 1976: $121 \mathrm{ff}) .{ }^{10}$ Die soziologische Perspektive muss also entsprechend erweitert werden, um die Visualität von Geld berücksichtigen zu können. Dazu werden im Folgenden die semiotische und die kunsthistorische Perspektive - die in vielerlei Hinsicht maßgeblich für die visuelle Soziologie sind - herangezogen.

Für die Semiotik fungieren Banknoten als Versinnlichung von Zahlungsoperationen, als deren konkrete Zeichen. Nach Peirce ist ein Zeichen die Korrelation von 1) Repräsentamen (Zeichenträger), 2) seinem (Referenz-)Objekt und 3) seiner Bedeutung (Interpretant). Die Verknüpfung eines Zeichenträgers (seien dies nun Worte, Bilder oder geistige Vorstellungen) mit einem Objekt führt zu einer „mental or behavioral interpretation of the sign“ (Nöth 2011: 302). ${ }^{11}$ Versuchen wir an dieser Stelle die Banknoten einmal semiotisch zu betrachten: Die Zeichenrelationen sind hier besonders komplex. Ein konkreter Geldschein (z.B. ein 50€Schein) ist Zeichen für eine abstrakte Banknote (z.B. die 50€-Note), welche ihrerseits ein Zeichen für den Wert von $50 €$ ist. Das materielle Geldzeichen ist somit der Signifikant (die Ausdrucksseite eines Zeichens). Was aber ist der Signifikat (die Inhaltsseite eines Zeichens), d.h. welche Objekte werden durch Geldzeichen repräsentiert? Im Grunde repräsentieren Geldzeichen ,den Wert, den Gegenstände und Dienstleistungen in einem bestimmten Wirtschaftssystem haben und der sich im Preis ausdrückt" (Komar 1991: 347). ${ }^{12}$ Sie verweisen also auf viele verschiedene andere Objekte und Zeichen und bringen diese (in Form von Zahlen, also

9 Die Konzeption von Geld als ein symbolisch generalisiertes Kommunikationsmedium ist durchaus kritisch zu sehen. Letztlich handelt es sich um eine kommunikationstheoretische Reformulierung des konventionellen ökonomischen Tauschmitteltheorems. Vergessen werden dabei u.a. die Herrschaftsund Ausbeutungsverhältnisse, die durch das Geld ermöglicht werden (vgl. Marx 1872; Ganßmann 1996; Deutschmann 2007). Worauf aus Platzgründen hier ebenfalls nicht eingegangen werden kann, ist der Aspekt der gesellschaftlichen Auswirkungen des Geldes (Vergesellschaftung, Kolonisierung der Lebenswelt, Geld als absolutes Mittel; vgl. Weber 1972; Habermas 1981; Simmel 1989).

10 Einige neuere, teils empirische Arbeiten zur sozialen Bedeutung des Geldes zeigen jedoch, dass die vielerlei Formen (Münzen, Banknoten, Kredit- und Debitkarten, Gutscheine, private komplementäre Währungen etc.), in denen Geld auftritt, ein jeweils unterschiedliches Geldhandeln bewirken können (Meier 2001; Ritzer 1995; Thiel 2012; Zelizer 1994). Auf die konkrete Visualität wird hier jedoch ebenfalls nicht eingegangen.

11 Aus Platzgründen werden nur ganz basale semiotische Aspekte des (Papier-)Geldes angeführt. Weder können alle Zeichenaspekte (etwa Ikon, Index, Symbol; vgl. hierzu Nöth 2000, 2011: 300ff) diskutiert noch unterschiedliche Ansätze verglichen werden (zu Geld bei Peirce und Saussure siehe etwa Ahonen 1989). Auch die Besonderheiten des Geldes im Vergleich zu anderen Zeichen (etwa seine hohe Performativität oder seine strikte Einbindung in gesellschaftlich-materielle Zwangsmechanismen, vgl. dazu Winkler 2004) müssen außen vor bleiben. Dies gilt ebenso für die - soziologisch teils anschlussfähigen - unterschiedlichen poststrukturalen Theoretiker, die sich mit Geld befassen, wie Lacan, Foucault, Deleuze, Derrida oder Baudrillard (Hörisch 1996: 72 f).

12 Der Wertbegriff bzw. dessen unterschiedliche Auffassungen (Kostenwert-, Nutzwert-, Arbeitswerttheorien) können hier nicht behandelt werden. Für eine auf Aktien bezogene Diskussion verschiedener Ansätze vgl. Athanassakis (2008: 14ff). 
Preisen) in Relation zueinander. Geld ist so gesehen ein „Metazeichen“ (Rotman 2000: 25). ${ }^{13}$ Seinen Sinn erhält es durch das Vertrauen auf die Einlösbarkeit bzw. Konvertierbarkeit. Geld ist ein Wertversprechen und die Materialität der Geldzeichen ermöglicht (ähnlich wie die Schrift für die Sprache) die Fixierung und Aufbewahrung von Werten. Diese Werte sind abstrakt und konkretisieren sich erst im Kaufvorgang. Einen unmittelbaren Objektbezug hat also nur die Zahl, die den Wert des Geldzeichens zum Ausdruck bringt. ${ }^{14}$ Dieser Objektbezug entsteht durch staatliche Institutionalisierung; er gilt aber nur solange, wie die Vereinbarung der Währungsgemeinschaft aufrechterhalten bzw. vom Staat garantiert wird (Komar 1991: 348). Besteht also die Bedeutung von Banknoten ausschließlich in dem funktionalen Element der Zahl? Wahrscheinlich nicht, denn die Worte und Bilder, die die Zahlen umgeben, haben ihrerseits eine bestimmte funktionale Aufgabe. Das Geld verweist in seiner Gestaltung nicht nur auf die Wirtschaft, es greift vielmehr semantisch in andere Sphären über. Diese funktionalen Übergriffe helfen den Wert des Geldscheines zu decken, indem sich der wertlose Papierzettel in außerökonomischen Systemen (wie Religion, Kunst und Liebe) symbolisch „refinanziert" (Hörisch 1996: 84). Von diesem Objektbezug (semantische Dimension) kann man beim Geld wie bei allen Zeichen noch den Subjektbezug (pragmatische Dimension), der auf den Geldbenutzer gerichtet ist und den Mittelbezug (syntaktische Dimension), der sich auf das Material des Geldzeichens richtet, unterscheiden.

Was das Verhältnis Geldzeichen - Benutzer (Subjektbezug) angeht, so ist dieser keinesfalls arbiträr. Zum einen aufgrund der Charakteristika der Geldzeichen selbst. Banknoten sind Inhaberzeichen mit Umlaufbestimmung. Sie lauten also nur auf den Inhaber, auch wenn dieser sie sich unrechtmäßig angeeignet hat; durch ihre Annahme ist eine Zahlung definitiv geleistet und jegliche Rückgriffmöglichkeit auf den Vorbesitzer ausgeschlossen; sie sind ferner zeitlich wertbeständig, d.h. ihre Umlaufzeit kann nur durch eine zentrale staatliche Maßnahme begrenzt werden (Komar 1991: 350). Zum anderen ist der Umgang mit bestimmten Geldformen ein Ausdruck kulturell habitualisierter Verhaltensweisen wie unterschiedlicher Bedeutungszuschreibungen, die ihrerseits auch durch die materielle Form des jeweiligen Geldzeichens geformt werden können.

Damit wären wir schon beim Mittelbezug: Geld als allgemeines Verständigungsmittel bedient sich im Falle der Banknoten des Papiers als Zeichenmaterie sowie sorgfältig geplanter optischer, akustischer und taktiler Merkmale, um die Teilwerte einer nationalen Währung zu repräsentieren. Dabei ist das Geldzeichen nie ein Einzelzeichen; es ist ,immer eine Kombination aus verschiedenen Signifikations- und Sekuritätsbestandteilen, ein Komplex aus Form, Farbe, Zahl, Buchstaben, natürlichen und geometrischen Figuren, offensichtlichen und versteckten, vorder- und hintergründigen Bestandteilen“" (Komar 1991: 352).

13 Das Edelmetall-Geld früherer Zeiten verwies auf sein ihm innewohnendes materielles Äquivalent (den Silber- / Goldwert). Im Übergang von der Edelmetalldeckung zum modernen, nur noch durch staatliches Wertversprechen gedeckten Fiat-Geld ist eine wesentliche Änderung der Struktur der Geldzeichen eingetreten. Das Geld verweist fortan auf die gesamten Güter und Guthaben einer Währungsregion und ist damit auch Zeichen der Kaufkraft innerhalb des Landes (Athanassakis 2008: 31). Dieser Übergang ging fließend vor sich, was man gut an den Vorgängern unseres Papiergeldes erkennen kann: Die Wechsel, Schuldscheine, Quittungen und Zahlungsanweisungen, die seit dem späten Mittelalter entstanden, waren zunächst inhärent deiktische, d.h. indexialische Zeichen. Diese Bezugnahme auf konkrete Personen, Orte und Zeiten findet sich auch auf frühen Banknoten, wo etwa noch der Name des Inhabers eingetragen war, auch wenn sie schon frei zirkulieren konnten. Es dauerte eine lange Zeit, bis der Schritt von einem deiktischen Versprechen mit seiner Einbettung in Daten, einem einzelnen Namen und einem Zeugenapparat hin zu einem entpersonalisierten, anonymen Inhaber vollzogen war (Rotman 2000: 85).

14 Nicht umsonst tauchen Zahlen so häufig auf Banknoten auf - auf der 20€-Note beispielsweise findet sich 14-mal die Zahl ,20` in unterschiedlichen Darstellungsweisen. 
Die eben angeführten Zeichenrelationen (Objekt-, Subjekt-, Mittelbezug) bieten interessante Anknüpfungspunkte für eine Soziologie des Geldes. Für eine Analyse der Visualität des Geldes bzw. einer darauf ausgerichteten systematisierenden Aufarbeitung des Forschungsstandes ist allerdings eine entsprechende Heuristik erforderlich. Für diese soll nun auf die Kunstgeschichte zurückgegriffen werden - schließlich hat diese die Fragen der Analyse und Interpretation von Bildern am intensivsten diskutiert. Zentral hier ist vor allem Panofskys Methode der Ikonologie. In Anlehnung an Aby Warburgs These, dass jedes Kunstwerk in den Gesamtzusammenhang der geistigen Welt eingebettet ist, in der es entstand, entwickelte Panofsky ein dreistufiges Verfahren, Bilder als Symbol weltanschaulicher Vorstellungen zu interpretieren (Panofsky 2006). Wie bislang üblich in der Ikonographie analysiert auch Panofsky zunächst, was (Semantik) auf welche Weise (Syntax) dargestellt ist. Mit seinem (dritten) Schritt, der ,ikonologischen Interpretation' geht er darüber hinaus. Er will mehr als eine Ikonographie im Sinne einer ,Bildniskunde' durchführen, die sich in einer Beschreibung und Auflistung der abgebildeten Personen, Themen und Motive erschöpft - er will Bilder als Ausdruck bestimmter kultureller, sozialer und historischer Hintergründe betrachten. Banknoten wären demgemäß ein Spiegel historischer Ereignisse und zeitgeschichtlicher Entwicklungen. Eines gilt es jedoch zu beachten: Man sollte Bilder respektive Kunstwerke nicht einfach zu simplen Dokumenten bzw. Illustrationen der Geistesgeschichte degradieren. Bilder haben ihre ganz spezielle Eigenlogik und Eigensinnigkeit. Die Frage, wie man dieser gerecht werden kann, wurde von verschiedensten Seiten behandelt wie etwa der Semiotik (Barthes, Eco), der Philosophie (Foucault) oder der Kunstgeschichte (Imdahl) (Bohnsack 2006: 46). Max Imdahl etwa erweitert entsprechend Panofskys Analyse des inhaltlichen Bildsinnes mit der des formalen Bildsinnes (Syntaktik), d.h. der Art und Weise, wie etwas durch Kompositionen und Formen dargestellt wird (Imdahl 1995). ${ }^{15}$ Für die Analyse von Banknoten wichtig scheint also, sie als Bild sowohl als Symbol bestimmter weltanschaulicher Vorstellung (Panofsky) als auch in ihrer Eigenlogik zu interpretieren.

Ein weiterer Aspekt kommt hinzu: Die Bildwissenschaften betonen immer wieder die Wichtigkeit des Kontextes der Produktion. Am Beispiel der Fotografie wird dies vielleicht deutlich: Diese bildet ja nicht einfach die Realität ab, sondern es spielt eine eminent wichtige Rolle, welcher Fotograf wie und aufgrund welcher Motive bestimmte Entscheidungen hinsichtlich Bildausschnitt, Standpunkt, Belichtung etc. trifft. Bei Banknoten ist dies besonders komplex. Zum einen erfolgt hier keine simple Abbildung (d.h. der Zeichen-Objekt-Bezug ist nicht eindeutig); zum anderen sind Geldscheine keine singulären Kunstwerke, sondern Erzeugnisse einer hochspezialisierten Massenfabrikation.

Ebenfalls muss die Ebene der Rezeption bzw. der Rezipienten beachtet werden. Barthes fragte einmal: „Wie gelangt der Sinn in das Bild?“ (1990: 28) und grenzte sich damit von substanzialistischen Auffassung ab, nach denen der Sinn fest und unveränderlich im Bild angelegt wäre und ihm von den Rezipienten in eindeutiger und gleichbleibender Weise ,entnommen' werden könnte. Stattdessen sind Bilder offen für unterschiedliche Sinnzuschreibungen, was zu einer Pluralität der Sinnbildungen führen kann (Michel 2007: 93). Barthes bezeichnet das als „Polysemie“ (1990: 34). Die Rezipienten können sogar ,oppositionelle“ Lesarten entwickeln und sich damit etwaigen Beeinflussungsabsichten der Bild- / Medienproduzenten entziehen (Michel 2007: 95). Allerdings wird nicht jede beliebige Sinnzuschreibung durch das Bild gedeckt; die „Logik des Signifikanten“ (Eco 1994: 163) setzt der Interpretation

15 In Imdahls Terminologie gibt es zum einen das, wiedererkennende Sehen', das beschreibt, was gemeint und gezeigt wird. Dies berücksichtigt den inhaltlichen Bildsinn, also die Semantik des Bildes und entspricht Panofskys Interpretationsmethode. Davon unterscheidet er das , sehende Sehen', also die Art und Weise wie etwas dargestellt ist. Dies richtet sich auf den formalen Bildsinn, die Syntaktik des Bildes (was in etwa der formalen Interpretationsweise wie sie etwa von Konrad Fiedler propagiert wird entspricht) (Waldenfels 2006: 234). 
gewisse Grenzen. Hinzu kommen, dass kollektive und präreflexive Prägungen (etwa der Habitus) der Rezipienten den aktiven Beitrag zur Sinnbildung beeinflussen (Michel 2007: 95).

Sensibilisiert durch die von der Semiotik proklamierten komplexen Zeichenrelationen sollen nun im Folgenden die bisherigen Forschungsergebnisse in diese der Bildwissenschaften entnommenen Heuristik (Bild(sinn), Produktionskontext, Rezeption) eingeordnet werden. Dies dient abschließend einer Erweiterung der (geld-)soziologischen Perspektive um den Aspekt des Visuellen.

\section{Aspekte der Visualität von Banknoten}

Die Gestaltung des Gegenstandes, Geld' ist etwas Besonderes. Im Unterschied zu anderen Gebrauchsgegenständen ist Geld nicht an eine bestimmte Gegenständlichkeit gebunden.

„,Während sie bei diesen funktional auf den Gebrauch bezogen bleibt, indem ein Stuhl nur dann schön ist, wenn er als Stuhl, nämlich als Sitzmöbel, schön ist, ist die Schönheit einer Münze [oder eines Geldscheins; C.T.] letztlich unabhängig von ihrem Gebrauch. "(Gabriel 2002: 42)

Das bedeutet allerdings nicht, dass die Gestaltung von Banknoten beliebig wäre. Im Gegenteil. Eine Vielzahl an Aspekten bestimmt die konkrete Visualität des Geldes.

\subsection{Der Bildsinn von Banknoten}

Betrachten wir einmal Banknoten im Allgemeinen. Es handelt sich hier um rechteckige, nicht übermäßig große Papierstücke, die mit diversen gestalterischen Merkmalen versehen sind: Wertangabe und Währungsbezeichnung; Geldscheinart; Ausgabe- oder Einlösestelle; Ortsangaben; Zeitangabe; Nummerierung oder Serienbezeichnung; Unterschriften; Strafsatz (Pick 1992: 38). ${ }^{16}$ Neben diesen ,funktionalen“ Aspekten findet sich vielfältiger Bildschmuck auf den Scheinen: Rahmen, Vignetten, Guillochen und Schraffuren, Porträts sowie Darstellungen von Tieren, Pflanzen oder Gebäuden. So unterschiedlich die einzelnen Banknoten auch wirken, so erscheinen sie doch immer als Geld. Offenbar gibt es ein gestalterisches Muster, das uns erlaubt, auch eine noch nie gesehene Fremdwährung auf Anhieb als Geld zu identifizieren, etwa durch eine charakteristische formale Komposition. ${ }^{17}$ Da es leider keine Studien über den formalen Bildsinn von Banknoten gibt, wenden wir uns an dieser Stelle ihrem inhaltlichen Bildsinn (bzw. dessen ikonologischer Interpretation) zu. Also: Welche Motive und Themen sind abgebildet und was sagen diese über die jeweilige Epoche aus?

Die ersten Banknoten (17. / 18. Jahrhundert) wirken wie Urkunden oder Verträge - auf ihnen findet sich nur Text, gelegentlich ein Rahmen und wenige Ornamente (Wertvignetten oder Schmuckbuchstaben). Man war bestrebt, den Noten einen Dokumentenstil zu geben und hielt jeden Schmuck für unseriös. Mit der Entwicklung der Drucktechnik im 18. und 19. Jahrhundert begann die Hinzunahme von Bildschmuck (oft wurden die auf Münzen verwendeten Adelswappen auf die Scheine übertragen) (Pick 1992: 39 f). Seit den Assignaten (dem während der französischen Revolution verwendeten Papiergeld) kam die Darstellung antiker Figuren in

16 Nicht immer sind alle Angaben zu finden - manchmal fehlt sogar die Wertbezeichnung, z.B. beim Kartongeld von Surinam (1816-1828) richtete sich der Wert nach dem Durchmesser des Kartons (Pick 1992: 38).

17 Darunter fallen beispielsweise bestimmte planimetrische Kompositionen wie die collageähnliche Anordnung der verschiedenen Elemente, bei der etwa die Porträts leicht seitlich versetzt sind oder eine spezifische szenische Choreographie, bei der die unterschiedlichen abgebildeten Teile in einen farblich und stilistisch angeglichenen Gesamtzusammenhang eingebunden werden. Natürlich verändern sich solche Formalstrukturen auch. So hat etwa die Größe der Porträts in den letzten Jahrzehnten deutlich zugenommen (Heij 2012: 46). 
Mode. Meist waren dies Fruchtbarkeits- und Glücksgöttinnen wie Ceres oder Fortuna. Es scheint, als sollte durch diese Anleihen an altbekannte und damit legitimationsstiftende Symbole (antike Götter, Tempel und mythologische Tiere) die Geltung des Geldes auch nach der Umstellung von Substanz- auf Funktionswert sichergestellt werden (Miklautz 2005: 52).

„So ist die ,Ornamentik' der Banknoten nicht nur eine Legitimationszeichnung des jungen Kapitalismus, sondern das Geld in Form von ,Schein' muß selbst mit Geistern aufgeladen werden, die das Glück sind, die Fruchtbarkeit, der Wohlstand, junge Frauen allemal, Mütter- und Segensgöttinnen etc. " (Priddat 2003: 19)

Man darf annehmen, dass dieser Religionsbezug die Akzeptanz des Papiergeldes durchaus förderte. Ende des 19. Jahrhunderts tauchen Symbole der Industrialisierung (Zahnräder, Werkzeuge etc.) auf und verbinden sich in manchmal skurriler Art mit der antiken Sagenwelt beispielsweise wenn ein halbnackter Hermes auf einem Elektromotor sitzt (abgebildet auf dem 1-Boliviano-Schein, der ab Mai 1911 herausgegeben wurde). Zur selben Zeit vollzieht sich ein Wandel vom bürgerlichen Fruchtbarkeitsideal zum staatlichen Hoheitszeichen. Die Länder selbst werden personifiziert als mythische weibliche Staatssymbole in wogenden Roben (Britannie, Svea, Germania, Austria, Italia, Roma, Lusitania etc.). Dieses Göttinnenpersonal wird ab etwa 1920 sukzessive gegen Menschen ausgewechselt - ,an die Stelle der allegorischen Religion tritt das kollektive Gedächtnis der Gesellschaften“"(Priddat 2003: 29). In den nächsten dreißig Jahren finden sich dementsprechend immer häufiger materialistische Beschreibungen des Staates (echte historische Staatsmänner) oder der Gesellschaft (Symbole für Klassen etc.) (Brion / Moreau 2001: 34ff). ${ }^{18}$ Ab ca. 1950 wechselt die Darstellung erneut. Nun dominieren nicht-staatliche Vertreter der hohen Künste (Verdi, Strauss, Swift etc.). Diesen treten ab ca. 1980 weitere, teils weniger gewichtige Akteure zur Seite, beispielsweise Autoren für Kinderbücher (Saint-Exupéry) oder Sportler. Auch Frauen, die seit dem Verschwinden der antiken Göttinnen eher selten abgebildet wurden, finden sich nun wieder häufiger (Hymans 2005: 320).

Eine der wenigen Studien, die diesen Wandel von Banknoten im Zeitverlauf empirisch analysiert, ${ }^{19}$ kommt zu dem Schluss, dass sich darin der Einfluss bestimmter ,cultural shifts ' niederschlägt: Zum einen der Wandel der Legitimationen vom Staat (mythisch-allegorische Figuren) über die Gesellschaft (Repräsentanten sozialer Gruppen / Klassen) hin zu Individuen (bspw. Künstler). Zum anderen eine Änderung der Lebensziele von der Ergebenheit zu Staat und Vaterland über materiellen Erfolg hin zu einem postmateriellen Fokus auf Lebensqualität und künstlerisch-wissenschaftliches Streben (Hymans 2004: 8 f). Trotz aller methodologischer Einwände gegen diese etwas oberflächliche Analyse scheint es gewisse Trends bei der Gestaltung der europäischen Banknoten zu geben. Sie verändern sich zwar stark im Zeitverlauf, sind aber zu gleichen Zeitpunkten jeweils recht ähnlich. Natürlich muss man dies differenziert betrachten - so haben sich in einigen Ländern sehr charakteristische Notentypen entwickelt (bspw. der Blaudruck der Banque de France oder die von der Deutschen Reichsbank nach dem ersten Weltkrieg herausgegebenen Noten mit Kopfbildnissen nach berühmten Gemälden). Dennoch scheint es grundsätzliche Muster hinsichtlich Inhalt und Darstellung zu geben, die Staaten auch voneinander übernehmen. Der Yen beispielsweise bildet entsprechend des euro-

18 Ein Beispiel ist die ,Holbein-Serie“ der deutschen Reichsbank von 1928. Auf den neuen Banknoten erscheinen etwa Albrecht Daniel Thaer (Begründer der rationellen Landwirtschaft); Werner von Siemens (Industrieller) und David Hansemann (Finanzpolitiker). Wegen der unpolitischen und wirtschaftsfreundlichen Motivwahl - die in scharfem Kontrast zur Kunstszene der Weimarer Republik stand - nannte man die neue Notenreihe bald die „Wirtschaftsserie“ (Weimer 1994: 208).

19 Konkret durchgeführt wurde hier eine quantitative Bildanalyse von 1368 Banknoten aus 15 europäischen Ländern Dabei wurden die abgebildeten Personen verschiedenen Kategorien entlang der Achse ,Akteur' (Staat-Gesellschaft-Individuum) sowie der Achse ,Ziel' (Traditional-Materialistisch-Postmaterialistisch) zugeordnet (Hymans 2004). 
päischen Musters ab 1980 ebenfalls nichtstaatliche Individuen (Philosoph, Schriftsteller, Bakteriologen) sowie postmaterialistische Ziele ab (Hymans 2005: 323ff).

Zusammenfassend lässt sich festhalten: Die Gestaltung von Geldscheinen wandelt sich mit der Zeit. Die ersten schmucklosen Vertragsdokumente werden bald motivreich ausgestaltet. Dabei sagen die vielfältigen Abbildungen von Göttinnen und Staatssymbolen, Herrschern und gesellschaftlichen Klassen, Künstlern und Wissenschaftlern ganz im Sinne Panofskys viel über die jeweilige Gesellschaft aus. Beispielsweise lässt sich hier der Grad der Staatlichkeit ablesen - die sich erst konstituierenden Nationalstaaten des 18. und 19. Jahrhunderts mussten noch legitimationsstiftende Anleihen bei der antiken Mythologie nehmen. Später dominierten durchaus nationalistische Darstellungen von Herrschern und gesellschaftlichen Klassen, auf die in der fortgeschrittenen Staatlichkeit zugunsten von Künstlern verzichtet werden konnte. So gesehen sind Banknoten ein Anschauungsunterricht der geschichtlichen, kulturellen und wirtschaftlichen Entwicklung vieler Länder (Pick 1992: 39ff).

Aber: So sehr Banknoten auch die kulturelle und historische Einbettung in eine Gesellschaft widerspiegeln, so sehr finden sich auch weltweit übergreifende Muster, oft hinsichtlich der Art der Darstellung (formaler Bildsinn). Im Zeitverlauf lassen sich die Entstehung solcher charakteristischer Darstellungsweisen und ikonologischer Versatzstücke beobachten, die von Banknoten aller Länder übernommen werden. Eines der ältesten ist etwa die Unterschrift, die bereits auf den Wechseln bzw. Schuldscheinen und heute auf ziemlich jeder Nationalwährung der Welt abgebildet ist (beim Euro etwa die Unterschrift des EZB-Präsidenten). Andere Gemeinsamkeiten beziehen sich auf die planimetrische Komposition, auf bestimmte Rahmen, Vignetten oder charakteristische Schmuckelemente. Während einige dieser Gestaltungselemente im Zeitverlauf verschwinden, bleiben andere bestehen. Warum dies so ist, wurde noch nicht untersucht. Möglicherweise gibt es aber so etwas wie ein globales, sich im Zeitverlauf wandelndes Muster der Darstellung von Geld.

\subsection{Der Produktionskontext}

Banknoten sind ein hochspezialisiertes Massenprodukt und entsprechend komplex ist der dahinterstehende Produktionskontext. Um nun verstehen zu können, inwiefern dieser einen Einfluss auf die konkrete Gestaltung von Banknoten hat, muss beachtet werden, welche Anforderungen überhaupt erfüllt werden müssen (Gestaltungsaspekte), wie diese umgesetzt werden (Produktionsprozess) und wer tatsächlich diesen Prozess der Konstruktion und Repräsentation kontrolliert (Gestaltungsvorgaben) (Penrose 2011: 432).

\subsubsection{Gestaltungsaspekte}

Warum werden Banknoten überhaupt in der Regel alle zehn bis 15 Jahre neu gestaltet? ${ }^{20}$ Es gibt eine Reihe von Gründen: Eine Währungsreform wird durchgeführt; aufgrund von Inflation sind neue Denominationen erforderlich; die Öffentlichkeit akzeptiert die Banknoten nicht; die Haltbarkeit ist zu gering; es wurden Fehler (in der Beschriftung) festgestellt; die Handhabung soll verbessert werden (etwa für Sehbehinderte); die logistischen Anforderungen haben sich verändert; die Leitung der Zentralbank hat gewechselt (was eine neue Unterschrift nötig macht); man verfolgt bestimmte propagandistische Ziele (Heij 2010 a: 9). Der wohl wichtigste Grund ist sicherlich die Fälschungssicherheit. Aufgrund des niedrigen Materialwerts wurde Papiergeld immer schon gefälscht. Übrigens nicht nur von ,privaten' Kriminellen, sondern auch von Staaten, getreu des Mottos von Lenin: ,Wer eine Gesellschaft zerstören will, muss

20 Laut US Treasury sollte alle 7 bis 10 Jahre eine neue Banknoten-Serie herausgegeben werden (Heij 2010 a: 17). 
ihre Währung ruinieren'. Dies hat schon Napoleon mit der Fälschung der österreichischen und russischen Währungen versucht (Pick 1992: 19) oder die Nazis beim britischen Pfund (Walburg 2011: 70). ${ }^{21}$ Die Fälschungsproblematik verschärfte sich ab den 1980er Jahren mit dem Aufkommen der digitalen Repro-Technologie. Das ,Digifeiting“ ermöglichte es auf die bislang aufwendige Ausstattung (Werkstatt, Druckmaschine etc.) zu verzichten und mit einem simplen Kopierer bzw. Scanner und Farbdrucker gefälschte Scheine herzustellen. Moderne Banknoten sind deswegen mit einer ganzen Reihe an Sicherheitsmerkmalen versehen. Diese müssen so gestaltet sein, dass auch der Laie eine Fälschung erkennt - etwa durch sogenannte, trigger features', die es ermöglichen, eine Banknote aufgrund bestimmter haptischer, akustischer und optischer Merkmale zu ,erfühlen' (Heij 2010 a: 11). Ein weiterer wichtiger Aspekt bei der Gestaltung von neuen Banknoten betrifft ihre Identität. Hier gilt es diverse Punkte festzulegen: Soll das vorige Design erweitert werden oder gibt es ein komplettes Neudesign? Welche der neu entstandenen drucktechnischen Innovationen übernimmt man? Wird die Öffentlichkeit in die Gestaltung eingebunden oder nicht? Welche Emotionen, welche nationalen Gefühle sollen adressiert werden? Orientiert man sich an den Banknoten anderer Länder? Wichtig bei der Auswahl der Bild-Motive ist zudem der Aspekt der Werterkennung: Tragen die gewählten Bild-Motive dazu bei, Banknoten sowie ihre unterschiedlichen Denominationen auf Anhieb zu erkennen und auch „,wertvoll“ erscheinen zu lassen? ${ }^{22}$ Ein ganz pragmatischer, weil kostenrelevanter Aspekt ist die Haltbarkeit: Viele moderne Banknoten verwenden deswegen neuentwickelte Materialien (Polymer statt Papier) und drucktechnische Finessen (etwa das von De La Rue patentierte Cornerstone-Verfahren, das ein Umknicken und Abreißen von Ecken erschwert) (Heij 2010 a: 11). Schon während des Gestaltungsprozesses muss man sich zudem überlegen, wie die neue Währung kommuniziert werden sollen: Hier bedarf es eines verständlichen Grundthemas der Banknoten sowie einer Kombination von einfacher öffentlicher Erkennung und hoher Fälschungssicherheit. Sämtliche der genannten Elemente muss der jeweilige Designer in sein grafisches Konzept implementieren. Wie die Deutsche Bundesbank feststellt,

„,zählt die Gestaltung von Banknoten zu den schwierigsten Aufgaben, die an einen Grafikdesigner gestellt werden können. Eine ungewöhnlich hohe Anzahl vorgegebener graphischer Elemente ist auf relativ kleiner Fläche übersichtlich anzuordnen. Dabei engen sicherungs-, fertigungs-, anwendungs- und bearbeitungstechnische Forderungen an eine moderne Banknote den kreativen Spielraum des Entwerfers empfindlich ein. [...] Bei der optischen Gestaltung ist zu berücksichtigen, dass die Banknote eine Drucksache der denkbar größten Öffentlichkeit ist. Als gültiges Zahlungsmittel ist sie Gegenstand des täglichen Gebrauchs für jedermann, als Werbeträger sollte sie ein Spiegelbild der Werte sein, die sie repräsentiert, wie Stabilität, Kontinuität und Krisenfestigkeit. [...] Daher wird die Banknote gelegentlich auch als Visitenkarte eines Staates bezeichnet" (Deutsche Bundesbank 2010).

21 Aus Angst vor politisch motivierten Fälschungen produzierte die Deutsche Bundesbank Anfang der 1960er Jahre eine nach ihrem Gestalter genannte Ersatzwährung, die „Bittrof-Serie“ - „für alle Fälle“ (Walburg 2011: 69), wie es hieß. Insgesamt wurden von 1963 bis 197425 Milliarden DM gedruckt. Mit der Einführung der letzten DM-Serie wurde die Idee einer Ersatzwährung gestrichen und sämtliche Scheine 1988 / 1989 geschreddert (Walburg 2011).

22 Einige Autoren behaupten, die Darstellung menschlicher Gesichter auf Banknoten mache diese fälschungssicherer. Da Menschen sehr gute Fähigkeiten zur Gesichtserkennung haben, würden schon minimalste Abweichungen bei Fälschungen auffallen (Unwin / Hewitt 2001: 1013; kritisch dazu Pointon 1998: 237). Dazu passend wurde in einer Studie ermittelt, dass wenn die Hauptbilder auf dem Euro (Brücken, Tore und Fenster) ausgetauscht werden, dies $80 \%$ der Befragten nicht auffiel. Zumindest für die Wertkennung der einzelnen Denominationen waren die Bilder der Euroscheine anscheinend eine schlechte Wahl (Heij 2010 a: 10). 


\subsubsection{Der Produktionsprozess}

Die Banknotendrucker sind weit mehr als ein rein ausführendes Organ. Seit Beginn ist die Gestaltung von Banknoten stark beeinflusst von der Drucktechnik und der dahinterstehenden Branche des Hochsicherheitsdrucks. Diese bringt nicht nur zentrale Gestaltungselemente hervor und verbreitet sie global, sondern hat unser modernes Papiergeld überhaupt erst ermöglicht. Robertson (2005) zeigt auf, wie mittels der mechanischen Reproduktion und einer technischzeichnerischen Ästhetik überhaupt erst Vertrauen in Papiergeld geschaffen wurde. Im England des Jahres 1800 herrschte ein tiefes Misstrauen gegenüber dem Papiergeld. Es wurde als subversiv wahrgenommen, da sowohl der Amerikanische Bürgerkrieg als auch die Französische Revolution mittels der Ausgabe von Banknoten finanziert wurden; wegen der häufigen Fälschungen und den diversen staatlichen (Über-)Emissionen galt es als wenig vertrauenswürdig und nicht wertbeständig. Bereits 50 Jahre später wurde es dank rapider Fortschritte in der Drucktechnik allgemein akzeptiert. ${ }^{23}$ Diese ermöglichten erstmals eine exakte mechanische Reproduktion. Durch die komplexe mechanisch-technische Linienführung (feinste Details durch Stahlstich) und den Einsatz von Guillochen (komplizierte wellenförmige Linienmuster) war die Produktion von Banknoten nicht mehr handwerklich, sondern nur noch mit hohem Aufwand maschinell zu leisten (Robertson 2005: 39). Indem die (damals weit verbreitete) visuelle Rhetorik des mittels Stahlstich umgesetzten technischen Zeichnens aufgegriffen wurde, erschien die Banknote selbst als Produkt des industriellen Prozesses (Robertson 2005: 35). Mit der Verwendung dieser maschinellen Ästhetik stieg das Vertrauen in das Papiergeld und eine charakteristische Ornamentik entstand, die auch heute noch maßgeblich für den Wertpapierdruck ist.

Nun war damit das Problem der Fälschung noch lange nicht beseitigt. Auch das beste Verfahren kann irgendwann kopiert werden. Die Druckereien waren und sind deswegen gezwungen, durch ständige Innovationen und den Einbau von Sicherheitsmerkmalen der Drucktechnik ihrer Zeit immer einige Schritte voraus zu sein. Im 19. und beginnenden 20. Jahrhundert führte das zur Entstehung einiger hochqualifizierter Spezialfirmen (teils privat, teils staatlich). Diese setzten die Standards, was zu einer gewissen Uniformität der Banknoten weltweit führte. Als die Japaner beispielsweise zu Beginn der Meiji-Ära 1868 neue Banknoten planten, orderten sie ein Hochsicherheits-Notendruck-System aus Deutschland; die Gravierung der Druckplatten wurde von einem Italiener ausgeführt. Die Folge dieses intensiven Technologietransfers waren, westlich' gestaltete Banknoten (Uemura 2007: 129ff). Die Schweden wiederum orientierten sich maßgeblich an der englischen Drucktechnik (Nathorst-Böös 1970). Andere Staaten ließen gar die komplette Währung von ausländischen Notendruckereien anfertigen (Helleiner 2003: 103).

Heute verfügen die meisten Länder über eigene Staatsdruckereien; bekannte sind etwa die deutsche Bundesdruckerei, das amerikanische Bureau of Engraving and Printing, die Druckerei der Banque de France oder die Österreichische Staatsdruckerei. Etliche von diesen produzieren auch Banknoten anderer Länder (so drucken etwa die Österreicher den kroatischen Kuna). Daneben gibt es noch einige Privatfirmen, in Deutschland Giesecke \& Devrient, in England De La Rue, in den USA die American Banknote Company; in den Niederlanden Joh. Enschedé und in der Schweiz Orell Füssli (Pick 1992: 85 f). Diese drucken ebenfalls - zumindest in Teilen - verschiedene Nationalwährungen sowie diverse Hochsicherheitsdokumente

$23 \mathrm{Zu}$ jener Zeit wurden eine Vielzahl drucktechnischer Innovationen geschaffen: Jacob Perkins erfand 1819 das Molettierverfahren der Siderographie, wodurch sich die Stahlstichplatten unbegrenzt und vor allem identisch vervielfältigen ließen. Jakob Degen entwickelte um 1816 das Guillochiergerät und damit ein fälschungssicheres Verfahren zum beidseitigen und mehrfachen Drucken von Banknoten. Weitere Erfindungen ermöglichten die Verwendung mehrerer Druckplatten beim Druck einer Note, was ebenfalls die Fälschungssicherheit erhöhte (Robertson 2005: 36). 
(Schecks, Wertpapiere, Pässe, Ausweise, Eintrittskarten, Flugtickets, Bankkarten etc.). ${ }^{24}$ Momentan ist der Druck von Banknoten zwar weitestgehend staatlich, aber es ist eine Tendenz festzustellen, die Banknotenproduktion zu delegieren (an den privaten Sektor, etwa im United Kingdom oder Schweden), zu privatisieren (Gründung von Tochtergesellschaften, etwa in Australien oder Bulgarien) oder zu rationalisieren (Zusammenlegen von Druck und Distribution, etwa in Kolumbien) (Galán / Sarmiento 2008: 218ff). Es bleibt abzuwarten, welche Auswirkungen auf die Gestaltung sich daraus ergeben.

\subsubsection{Die Gestaltungsvorgaben}

Trotz aller zu beachtenden Gestaltungsaspekte, trotz des Einflusses des Produktionsprozesses könnte man annehmen, dass zumindest die groben Gestaltungsvorgaben von klar umrissenen staatlichen Akteuren formuliert werden. Es scheint allerdings, dass die staatliche Einflussnahme auf die Gestaltung zum Zeitpunkt der Staatsbildung am intensivsten war. Damals wurde das Papiergeld ausdrücklich in den Dienst des Aufbaus der nationalen Identität gestellt und ein entsprechendes symbolisches Repertoire entworfen. War der Staat erst etabliert, so schwand sein Einfluss und andere Akteure (Zentralbanken, Designer etc.) übernahmen die Definition von gestalterischen Leitlinien (Penrose 2011). Bei den meisten modernen Währungen ist das Banknotendesign ein komplexer und teils undurchsichtiger Prozess. Es werden zwar Gestaltungswettbewerbe durchgeführt, die Entscheidungen der Jurys sind jedoch nicht selten nebulös und die dafür verantwortlichen Komitees häufig intransparent zusammengesetzt. Manchmal werden Währungen von Politikern quasi im Alleingang konzipiert (Wallach 2011); anderswo geben Currency Boards den Ton an (Mwangi 2002). Fast immer redet eine Vielzahl an Leuten mit (Hewitt 2001). Darüber hinaus beeinflussen universelle Sicherheitsbedenken, internationale Stilkonventionen (Hymans 2005) oder ideologische Haltungen (Gilbert 1998, 1999) die Banknoten in Form und Inhalt. Dadurch ist die Konzeption ihrer Gestaltung keineswegs immer rational und zielgerichtet. Ein paar Beispiele mögen dies verdeutlichen:

Für die letzte Serie der DM wurde 1987 ein Gestaltungswettbewerb durchgeführt. Dafür wählte ein Gremium von drei Historikern aus einer Liste mit 80 Vorschlägen einige Persönlichkeiten aus der Deutschen Geschichte aus. Man verzichtete auf offensichtliche Größen wie Goethe oder Dürer, auch auf solche die konfessionell oder politisch als Provokation hätten aufgefasst werden können wie Karl Marx oder Martin Luther. Die Hälfte sollte zudem weiblich sein. Nach der Vorauswahl hatte man allerdings ein ästhetisches Problem: Die für den 100DM-Schein vorgesehene Naturforscherin Maria Sibylla Merian hatte krankheitsbedingt her-

$24 \mathrm{Zu}$ beachten ist außerdem, dass hinter den verschiedenen Druckereien eine entsprechende Zulieferindustrie steht, konkret die Hersteller von Druckmaschinen, Farben und Papier. Diese Branche ist so exklusiv wie verschwiegen. Es ist Markt ohne Marktpreise, da aus Gründen der Fälschungssicherheit Exklusivität zwingend, Kostensenkung durch Erhöhung der Produktion kaum möglich und die Zahl der Anbieter so eng begrenzt ist, dass schon fast von einem Monopol gesprochen werden kann. Im Bereich der Druckmaschinen maßgeblich dafür verantwortlich war Gualtiero Giori. Der Italiener entwickelte zusammen mit der deutschen Firma Koenig\&Bauer eine hochpräzise druckende Maschine für vielfarbigen Intaglio-Stichtiefdruck. Er revolutionierte damit nach dem zweiten Weltkrieg den Wertpapierdruckmarkt - inzwischen werden mehr als 90\% aller auf der Welt gedruckten Banknoten auf seinen Maschinen gefertigt (Bender 2004: 47). Im Bereich der Sicherheitsfarben für Banknoten kontrolliert Albert Amon und seine Farbenfabrik SICPA den Weltmarkt. Sie meistern die hohen technischen Herausforderungen an die chemische Echtheit und physikalische Beschaffenheit, die exakt standardisiert und reproduzierbar sein muss (damit Banknoten mittels Spektralanalyse auf Echtheit überprüfbar sind). Einige Zahlen verdeutlichen die Größe dieses Marktes: Weltweit ist eine Stückzahl von insgesamt 180 bis 190 Milliarden Banknoten im Umlauf; das jährliche Druckvolumen beläuft sich auf 85 bis 95 Milliarden Stück (auf private Druckereien entfallen ca. 8 Milliarden) (Bender 2004: $36 \mathrm{f})$. 
vorstehende Augen. Trotz eines recht idealisierten Bildes war sie dem Konsortium nicht hübsch genug. In der Folge wurde sie auf den weniger gebräuchlichen 500-DM-Schein , verbannt' und die (hübschere) Clara Schuhmann sollte fortan den 100-DM-Schein schmücken (Maack 2011). Auch anderen Ländern fällt die Auswahl schwer: Als die Banque de France Mitte der 1990er die Brüder Lumière auf dem 100-Franc-Schein abbilden wollten, gab es Proteste, weil diese während des Zweiten Weltkrieges das Vichy-Regime unterstützt haben. Paul Cézanne kam ins Gespräch, wurde aber abgelehnt, da er lebenslang Geld gehasst hat (Dilley 2000).

Ein Beispiel für den Einfluss der Künstler findet sich etwa auf niederländischen GuldenNoten (herausgegeben ab 1966). Der Designer Oxenaar ,schmuggelte' seinen Fingerabdruck in die Locken von Spinozas Haaren. Auf anderen Entwürfen brachte seinen Namen oder den Hasen seiner Freundin (in einem Wasserzeichen) unter. Dies verdeutlicht eines: Das Banknotendesign kann willkürlich, ad hoc, inkonsistent und sehr personalisiert sein (Penrose 2011: 432).

Zusammenfassend betrachtet hat der Produktionskontext große Auswirkungen auf die Gestaltung von Banknoten. Eine Vielzahl an Gestaltungselementen muss berücksichtigt und im Rahmen eines hochkomplexen technischen Prozesses umgesetzt werden. Dazu gilt es eine Schar an Beteiligten (Zentralbank, Projektgruppen, Grafikdesigner, Drucker, Graveuren, Zulieferern von Papier, Sicherheitsfäden, Metallfolien, Tinten, Fasern, Hologrammen etc.) zu koordinieren und diverse nationale und internationale, gesellschaftliche und institutionelle Rahmenbedingungen zu berücksichtigen. ${ }^{25}$ Dabei ziehen einmal gemachte Fehler massive Kosten und Vertrauensverluste nach sich (Heij 2010 b: 16). Dementsprechend häufig kommt es zu Verspätungen: Die Dänen emittierten ihre neue Banknote 2009 mit einer einjährigen Verspätung; der neue 100-US\$-Schein ist bereits zwei Jahre hinter der Planung; die Schweizer haben mittlerweile erneut den (für 2010 geplanten) Ausgabetermin von 2012 auf 2013 verschoben; die 2. Serie der Euro-Banknoten (ES2) mit neuen Sicherheitselementen hätte ursprünglich schon 2003 herauskommen sollen und wird ab 2013 sukzessive eingeführt.

\section{Die Rezeption von Banknoten}

Wie Banknoten (bzw. ihre Visualität) tatsächlich wahrgenommen werden, lässt sich sehr schwer beantworten. Sie sind ein so selbstverständlicher Bestandteil des alltäglichen Lebens, dass sie kaum beachtet, geschweige denn intensiv ,betrachtet' werden. Dementsprechend gibt es kaum Studien, die sich dieser methodologischen Herausforderung stellen. Im folgenden Abschnitt wird deswegen zunächst betrachtet, welche Deutungsvorgaben durch die konkrete Gestaltung gemacht werden, also welche Rezeption intendiert ist. Soweit möglich wird dann dargestellt, welche tatsächliche Rezeption daraus resultiert. ${ }^{26}$ In der bisherigen Forschung wurden einige Thesen entwickelt, welche ,Botschaften' durch das Papiergeld typischerweise vermittelt würden. Einige davon seien im Folgenden kurz aufgeführt.

25 Besonders schwierig war dies beim Euro. Nicht nur, dass die komplett unterschiedlichen Bargeldtraditionen der einzelnen Mitgliedsländer berücksichtigt werden mussten. Auch wollte von diesem größten Druckauftrag der Geschichte jede Nation ihren Teil abhaben. In der Folge waren 9 Papiermühlen, 15 Notendrucker und mehr als 20 Zulieferer an der Herstellung beteiligt - ein sicherheitstechnischer Alptraum. Auch der Druck selbst entspricht nicht modernen Sicherheitskriterien. Wegen des französischen Rollendrucks konnte nur einseitig (Vorderseite) ein Stichtiefdruck erfolgen; das Wasserzeichen ist sehr simpel; es gibt - je nach Herstellerland - Farbabweichungen usw. (Bender 2004: 248ff).

26 Um nun die ,intendierte Rezeption“ vom vorab dargestellten ,Bildsinn“ (wenn auch nicht komplett trennscharf) abzugrenzen, kann man festhalten, dass es in diesem Abschnitt um von bestimmten Akteuren intendierte Botschaften geht, während unter dem, Bildsinn` eher subtil wirkende historischgesellschaftliche Rahmungen abgehandelt werden. 


\subsection{Banknoten dienen dem Aufbau und der Stärkung einer nationalen Identität}

Wie im 2. Kapitel schon angeführt, hat das Papiergeld eine wichtige Rolle im Prozess der Entstehung von Nationalstaaten während des 19. Jahrhundert gespielt - auf einer funktionalen und einer (für die Gestaltung wichtigen) symbolischen Ebene.

In funktionaler Hinsicht konnte durch die Vereinheitlichung und Verstaatlichung der Währung ein nationaler Markt aufgebaut werden, was den wirtschaftlichen Austausch deutlich erleichterte (Helleiner 2003: 110). Daraus resultierte auch der Aufbau einer nationalen Identität (Gilbert / Helleiner 1999 b: 7). Voraussetzung dafür ist, dass häufig, effizient und breitgefächert kommuniziert wird. Eine nationale Währung ist wie eine universelle Sprache, die alle verstehen und alle sprechen wollen; sie macht ,aus Bauern Bürger' (Helleiner 2003: 112). Weiterhin verstärkt Papiergeld die Bindung an den Staat. Da es keinen intrinsischen Wert hat, müssen die Bürger auf das staatliche Wertversprechen vertrauen - im Gegensatz zum ,kosmopolitischen' Edelmetall. Ein ganz konkreter Machtaspekt kommt hinzu. Die einheitliche territoriale Währung verstärkt die staatliche Souveränität, denn private Münzanstalten waren aufgrund ihres hohen Einkommens (und des daraus resultierenden Einflusses) immer potenzielle politische Unruheherde (Helleiner 2003: 116). Kurzum: Das einheitliche und staatliche Papiergeld förderte nicht nur die wirtschaftliche Entwicklung, sondern stärkte auch den Nationalstaat, weswegen dieses Modell weltweit adaptiert wurde.

Genauso wichtig ist allerdings der symbolische Aspekt, der zu jener Zeit ganz bewusst eingesetzt wurde. Man verwendete die bildliche Gestaltung des Papiergeldes um nationale Ideen zu transportieren. Banknoten hatten damals eine viel größere Reichweite als alle anderen Medien und konnten zudem ihre symbolischen Inhalte auch an Analphabeten vermitteln. Durch ihre Allgegenwart und starke Verflechtung in das alltägliche Handeln können sie eine ,imagined community“ (Anderson 1993) aufbauen und im Sinne eines „,banal nationalism“ (Billig 1995) zu bestärken. ${ }^{27}$

Dementsprechend begannen viele Regierungen im späten 19. Jahrhundert systematisch nationalistische Motive auf ihrem Geld abzubilden (Helleiner 2003: 101). In den USA etwa versuchte man im Rahmen der Geldreform von 1863 ein Gefühl von Nationalität zu vermitteln, indem man wichtige Persönlichkeiten (Columbus, Franklin, Washington), historische Schlüsselereignisse (Unabhängigkeitserklärung, Taufe der Pocahontas, Kapitulation von General Burgoyne, Landung der Pilgerväter) und nationale Symbole (Flagge, Adler, Capitol) abbildete. $\mathrm{Ob}$ dies nun tatsächlich ein Nationalbewusstsein aufgebaut hat, lässt sich nicht mehr feststellen.

Es gibt allerdings ein aktuelles Beispiel, bei dem versucht wurde eine (supra-)nationale Identität symbolisch zu konstruieren - den Euro. ${ }^{28}$ Wie schafft man eine kollektive Identität für (derzeit 23) unterschiedliche Nationen? Gestalterisch wurde dies umgesetzt mittels des Bezugs auf gemeinsame Werthaltungen und (unverfängliche) historische Bezüge. Die Fenster und Tore auf der Vorderseite der Banknoten symbolisieren den europäischen Geist der Offenheit und Zusammenarbeit. Die Brücken auf der Rückseite betonen die Kooperation und Kommunikation zwischen Europa und dem Rest der Welt. Die keinem Land zuordenbaren Architekturstile stellen eine Verbindung zu einer gemeinsamen europäischen Tradition her

27 Dies meint: Banknoten werden aufgrund ihrer Omnipräsenz (wie auch Briefmarken oder Straßennamen) im Alltag kaum beachtet bzw. bewusst wahrgenommen. Dennoch ,infiltrieren' sie den Alltag still und leise mit bestimmten (nationalistischen) Ideen, die dann als ganz ,normal ' und , selbstverständlich“ betrachtet werden (Raento et al. 2004: 930). Wenn Bürger Papiergeld benutzen, bestätigen sie fortwährend die nationale Gemeinschaft, da sie in den Wert ,ihres' Geldes bzw. in den dahinter stehenden Nationalstaat vertrauen (Cohen 1998; Gilbert 1998: 76).

28 Exemplarische Studien über die Konstruktion, Einführung und Auswirkungen des Euros, etwa Hymans 2004, 2006; Kaelberer 2004, 2005; Raento et al. 2004; Risse 2003; Winter 2007. 
(und erlauben gleichzeitig unangenehme Aspekte der europäischen Vergangenheit wie Kriege oder Imperialismus zugunsten einer optimistischen und progressiven Vision von Europa vergessen zu machen) (Kaelberer 2004: 13). Gleichzeitig mussten bei der Einführung die ,jeweiligen nationalen Währungskulturen“ (Löffler 2010: 3) der beteiligten Nationen berücksichtigt werden. Schließlich bedeutete der Euro einen Bruch mit dem Prinzip ,eine Nation - ein Geld', das die letzten 100 Jahre kennzeichnete. Es zeigte sich nun, dass anscheinend eine wechselseitige Beziehung zwischen kollektiver Identität und der europäischen Währung besteht. Bestehende kollektive (nationale) Identitäten bestimmen großteils, wie wohl sich Bürger der verschiedenen Länder beim Umgang mit dem Euro fühlen (Risse 2003: 487). Italien beispielsweise nahm den Euro sehr enthusiastisch auf, Deutschland eher reserviert und England komplett ablehnend (Risse 2003: 495). ${ }^{29}$ Dennoch hat die Einführung des Euros offenbar die Identifikation der Bürger mit der EU und Europa gesteigert. „The Euro makes Europa real“ (Risse 2003: 487); er stellt eine sichtbare Verbindung zwischen Brüssel und dem alltäglichen Leben her. Solange es keinen europäischen Fernsehsender gibt, bleiben die Euro-Banknoten wohl das einzige Medium, durch das Europa konkret und greifbar in den Alltag der Menschen tritt. So gesehen vermittelt der Euro also ein zumindest diffuses Gefühl der „Europeanness“ (Kaelberer 2004: 17). Zusammenfassend betrachtet scheinen Banknoten also durchaus einen Beitrag zu kollektiver (nationaler und supranationaler) Identität zu leisten. ${ }^{30}$

Allerdings sind einige Punkte kritisch anzumerken: Bezüglich der Verbindung von Nation und Geld gilt es zu beachten, dass diese historisch gesehen ein relativ junges Phänomen ist. Vor weniger als 150 Jahren waren Geld und Nation keineswegs deckungsgleich. Weiterhin sind und waren Nationalwährungen nicht immer stabil genug, um als anhaltende Symbole der nationalen Identität zu funktionieren (Kaelberer 2004: 8). Und: Kann man überhaupt von der nationalen Identität sprechen? Identitäten sind nicht fix, sie sind im ständigen Wandel, vielfältig und teilweise diffus. Weiterhin stellt sich die Frage, ob Geld überhaupt ein angemessenes Werkzeug ist, um symbolisch Territorien bzw. Identitäten abzugrenzen und zu definieren. Geld ist möglicherweise zu abstrakt, zu wenig greifbar und konkret, um eine klare Abgrenzung zwischen ,Wir' und ,Ihr' zu schaffen (Kaelberer 2005: 294). Damit stellt sich auch die Frage, ob eine von den Gestaltern intendierte Rezeption auch tatsächlich im Alltag so erfolgt. Eine Studie zur Wahrnehmung der Euro-Banknoten ergab beispielsweise, dass eine überwiegende Mehrheit die historischen Gebäude als Kernaussage sieht. Die eigentliche Absicht (verschiedene Zeitalter; Symbolik der Offenheit und Verbundenheit) wird kaum erkannt (Heij 2012).

\subsection{Banknoten symbolisieren Macht- und Herrschaftsverhältnisse}

Jenseits der nationalen Identität vermitteln Geldscheine als Instrument der politischen Symbolik auch andere Botschaften. Sie spiegeln etwa Herrschaftsverhältnisse oder die Zielsetzung bestimmter Regimes wider.

Klassische Beispiele sind etwa die von Besatzungs- und Kolonialmächten herausgegebenen Banknoten. Indem die dortigen lokalen Währungen zwangsweise entfernt wurden und sämtliche offizielle Vorgänge (Verwaltungsangelegenheiten, Steuern und Abgaben) nur noch in

29 Dies hat viel mit den Währungserfahrungen der letzten Jahrzehnte zu tun. In etlichen Ländern schwächelte die Währung (Haushaltsschwierigkeiten und Inflationsprobleme) dauerhaft. Entsprechend war das jeweilige nationale Geld schon lange kein Symbol des nationalen Stolzes mehr, mit der Folge, dass diese Länder (Frankreich, Spanien, Portugal, Italien, Griechenland) den Euro freudiger erwarteten (Kaelberer 2005: 286). In Deutschland hingegen wurde die DM positiv assoziiert mit dem Wirtschaftswunder der Nachkriegszeit, mit Stabilität und Stärke.

30 Der Großteil der Forschung zur Visualität von Banknoten bearbeitet diese These. Exemplarische Studien wären: Blaazer 2002; Fuller 2008; Gilbert 1998; Helleiner 2003; Hymans 2004, 2005; Kaelberer 2004, 2005; Penrose 2011; Pointon 1998; Raento et al. 2004; Risse 2003; Wallach 2011. 
der neu eingeführten Kolonialwährung vollzogen werden konnten, ließ sich eine tiefgreifende Kontrolle ausüben (Ofonagoro 1979). Die Gestaltung der Banknoten diente dabei der Verbreitung kolonialer Ideologien und der Untermauerung des Herrschaftsanspruchs. Dabei wurden viele Gestaltungsaspekte der ursprünglichen Währungen aufgegriffen (vgl. etwa Mwangi 2002). Auch Besatzungsmächte arbeiteten ähnlich. Während der nationalsozialistischen Besetzung wurden die tschechoslowakischen Banknoten durch Protektoratsbanknoten ersetzt. Diese ähnelten der alten Währung, symbolisierten aber gleichzeitig subtil die Unterordnung unter die Naziherrschaft - so wurde etwa auf einigen Scheinen die früher abgebildete phrygische Mütze (ein Freiheitssymbol) durch einen Strohhut ersetzt. Der böhmische Löwe prangte weiterhin auf einigen Scheinen, allerdings ohne das Nationalemblem auf seiner Brust (Veselkova / Horvath 2011). Solche symbolisierten Herrschaftsverhältnisse finden sich übrigens nicht nur bei Fremdherrschern. Etliche Nationen vermitteln auf ihren Banknoten auch, welche Gruppen innerhalb einer Gesellschaft welche Positionen haben bzw. wie einzelne Teile einer Nation zueinander stehen. Ein Beispiel für letzteres sind die frühen englischen Banknoten. Auf diesen wurde die Beziehung der Teile des United Kingdom mittels der Anordnung allegorischer Figuren verdeutlicht: Britannia ruht in der Mitte, an ihrer Seite sitzt eine (jüngere und kleinere) Scotia und zu ihren Füßen kniet demütig die Hibernia (Blaazer 2002: 15). Wieder stellt sich die Frage, ob eine so intendierte Gestaltung tatsächlich subtile Wirkung entfaltet oder ob die Nutzer abweichende Lesarten entwickeln.

\subsection{Banknoten kommunizieren politische Haltungen nach außen}

Geldscheine haben immer auch Außenwirkung. Für ausländische Besucher sind sie oft die erste Gelegenheit, etwas über die nationale Identität eines Landes zu erfahren (Unwin / Hewitt 2001: 1009). Gleichzeitig werden beim Benutzen von Fremdwährungen Unterschiede und Grenzen zwischen Nationalstaaten markiert und bestärkt (Penrose 2011: 429).

Die Botschaften auf Banknoten richten sich nicht nur an die eigenen Bürger bzw. Besucher, sondern teils auch explizit an andere Staaten. Beispielsweise wurde während des britischen Mandats in Palästina das palästinensische Pfund als Symbol gegen die arabischen Ansprüche gestaltet und eingeführt (Wallach 2011). Die finnische Staatsbank wiederum wählte 1897 als Motiv für ihre 20-Finnmark-Noten ein Denkmal, das die Finnen dem russischen Zaren Alexander II im Zentrum von Helsinki errichtet hatten. Dies war eine mehr oder weniger versteckte Botschaft an den damals herrschenden Zaren Nikolaus II., die dem (russischen) Großherzogtum Finnland im Jahre 1809 durch Alexander eingeräumte Autonomie nicht anzutasten (Bender 2004: 20 f). Kaiser Wilhelm II. richtete seine Botschaft gleich an die ganze Welt. Seine 1908 herausgegebene 100-Mark-Note schmückt eine misstrauisch blickende Germania, die Hand drohend am Schwertgriff, während im Hintergrund eine Formation Schlachtschiffe kreuzt. Das deutsche Kaiserreich machte damit unmissverständlich klar, dass es zur zweitgrößten Seemacht der Welt aufgestiegen ist (Bender 2004: 21). Ein letztes Beispiel aus einer Studie sei noch genannt, da diese als eine der wenigen auch die tatsächliche Rezeption einbezieht: In einer komplexen ikonographische Analyse der Banknoten der neu entstandenen Nationalstaaten Zentral- und Osteuropas ab den 1990er arbeiten Unwin und Hewitt (2001) heraus, wie diese sich symbolisch von der einstigen Sowjetherrschaft emanzipierten. Dies geschah unter anderem durch häufige Bezüge auf die lange unterdrückte christliche Identität (mittels der Darstellung von Kirchen und Klöstern). Im Großen und Ganzen wurde dies von den Bürgern sehr positiv aufgenommen - mit einer Ausnahme: Die kroatische Währung sollte eigentlich ,kruna' (übersetzt: Krone) heißen. Da dies als ,ausländisch' klingende Bezeichnung 
wahrgenommen wurde, gab es massive Proteste. Der Name wurde daraufhin in ,kuna'31 geändert (Unwin / Hewitt 2001: 1008).

\subsection{Banknoten symbolisieren Vertrauen}

Die häufig kolportierte These des „Staat[es] als Pädagoge“ (Hymans 2004: 5) lässt sich auch umkehren. Geht man davon aus, dass die staatliche Legitimität fragil ist, so ist es unwahrscheinlich, dass der Staat versucht, mittels Banknoten seinen Bürgern bestimmte Werte und nationales Bewusstsein ,einzuimpfen'. Stattdessen versuchen Staaten ihre Legitimität zu steigern, indem sie via Gestaltung der Währung signalisieren, dass sie im Einklang mit dem ,Zeitgeist' sind (Hymans 2004: 8). Dies geschieht zum einen durch das Aufnehmen bestimmter Gestaltungstrends (vgl. Kapitel 4.1); zum anderen durch das Beibehalten bereits anerkannter historischer Bezüge und ikonographischer Versatzstücke.

Ersteres wird u.a. in der Benennung vieler Währungen offensichtlich: Der ,Franc' wurde 1795 eingeführt, um das Geldvertrauen nach dem Scheitern der Assignaten wiederherzustellen; sein Name stammt von einer legendären und sehr stabilen Goldmünze des 14. Jahrhunderts. Auch der im Zuge der englischen Münzreform 1817 geprägte Sovereign wurde nach einer mittelalterlichen Münze benannt (Helleiner 2003: 108). Viele südamerikanische Länder wiederum benannten ihre Währungen nach berühmten Persönlichkeiten: Vasco Núñez de Balboa (panamaischer Balboa), Christoph Kolumbus (Costa-Rica-Colón, El-Salvador-Colón), Simón Bolívar (venezolanischer Bolívar, bolivischer Boliviano), Antonio José de Sucre (ecuadorianischer Sucre).

Weiterhin scheint im Laufe der Zeit ein charakteristischer ikonographischer Kanon zu entstehen, der als solcher Vertrauen sichert und dementsprechend hartnäckig ist. Offenbar gilt bei der Gestaltung von Geld „Kontinuität als Ausdruck von Stabilität“ (Gabriel 2002: 41). Und dies unabhängig von historischen Ereignissen wie Kriegen, Währungsreformen, Regimewechsel oder ideologischen Richtungen. So hatten in Deutschland die 100er-Noten seit Ausgabe der ersten Reichsbanknoten 1876 bis zur Einführung des Euros immer eine blaue Farbe - selbst in der DDR (Pick 1992: 42). Die 1000er waren immer braun (Pick 1992: 46). ${ }^{32}$ In England wiederum ist die Vignette der Britannia seit Gründung der Bank of England 1694 auf allen englischen Banknoten abgebildet (Hutter 2007).

Solche Gestaltungselemente sind Teil der beim Geld eminent wichtigen ,vertrauensbildenden Maßnahmen“ (Gabriel 2002: 36). Obwohl sie für die Wirtschaftstheorie sogenannte ,weiche' Faktoren darstellen, sind sie nicht zu vernachlässigen. Historisch gewordene Währungs,Bilder' sind ebenso realitäts- und handlungsprägend wie etwa institutionell-normative Strukturen. Sie legitimieren die Institutionen durch ideelle Grundlagen und tradierte Gewissheiten (Löffler 2010: 7). Nicht ohne Grund ist die Kategorie des kulturell bedingten ,Währungsgefühls' mittlerweile feste Größe in ökonomischen Statistiken (etwa Eurobarometer - ,Verbundenheit zum Euro'). Mit den Worten des ehemaligen Bundesbankpräsidenten Hans Tietmeyer „credibility is the name of the game“ (zit. nach Löffler 2010: 23). Inwiefern allerdings die Gestaltung von Geldscheinen eine solche ,credibility' befördert oder aber erschwert, muss noch erforscht werden. Ein methodologisches Problem dabei ist, dass Geld aufgrund seiner starken Verflechtung in den Alltag kaum bewusst wahrgenommen wird. Eine Studie der niederländischen Nationalbank zeigte etwa auf, dass nach 27 (!) Jahren nur 14\% der Befragten

31 ,Kuna‘ bedeutet übersetzt Marder, was letztlich ein Rückgriff auf uralte Geldtraditionen ist. Denn Marderfelle wurden im Kroatien des 13. und 14. Jahrhunderts als Währung verwendet.

32 Trotz der Trennung nach dem Zweiten Weltkrieg blieb das Geld (sowohl Banknoten als auch Münzen) von BRD und DDR relativ ähnlich. Trotz unterschiedlicher politischer Systeme reflektierten die Münzen und Banknoten offenbar eine gemeinsame nationale Tradition (Klüßendorf 2007: 41). 
die Person auf der 10-Gulden-Note benennen konnten (richtige Antwort: der Maler Frans Hals) (Heij 2008: 28). Beim Euro wissen viele nicht einmal die korrekten Denominationen - nur $53 \%$ kennen den 200-Euro-Schein; 14\% sind gar überzeugt, es gäbe eine 250-Euro-Note und 19\% glauben an die 1000-Euro-Note. Angesichts dessen wundert es nicht, wenn ein Geldfälscher problemlos seine mit barbusigen Frauen geschmückten 1000-Eros(sic!)-Scheine an den Mann bringt (Spiegel 12.7.2012). Wie man sich denken kann, hat das gravierende Auswirkungen auf die Sicherheit. Die besten Sicherheitsmerkmale nutzen wenig, wenn durchschnittlich nur 1,7 bekannt sind (am bekanntesten sind Wasserzeichen, Sicherheitsfaden und das ,Gefühl' von Papier und Druck) (Heij 2002). Allerdings scheint es eine positive Korrelation zwischen der Wahrnehmung einer Banknote (als ,schön“ oder ,hässlich') und der Kenntnis ihrer Sicherheitsmerkmale, Bilder und Textelemente zu geben (Heij 2002). ${ }^{33}$ Insgesamt erscheint eine eingehende Forschung zur tatsächlichen Rezeption von Banknoten deshalb relevant, weil sich so auch ,Fehler' in der Gestaltung vermeiden lassen. Ein Beispiel hierfür ist die etwas dumpfe Farbgebung des Euros, die dazu führt, dass gefälschte Scheine häufig als ,echt' erscheinen, weil sie einen helleren Farbton haben (Heij 2010 b: 16).

\section{Fazit und (methodischer) Ausblick}

Was lässt sich nun aus dem bisher Dargestellten aus soziologischer Sicht folgern? Meines Ermessens nach finden sich in der ganzen Vielfalt drei dezidiert soziologische Frage- bzw. Themenkomplexe:

Der erste zielt auf die Rolle, die Visualität für das symbolisch generalisierte Kommunikationsmedium Geld (zumindest in seiner konkreten Erscheinungsform als Banknote) spielt. Betrachtet man Banknoten hinsichtlich ihres Bildsinns, so zeigt sich ein starker Wandel der Motive und Themen: von Vertragsornamenten über antike Götter hin zu Staatssymbolen, von Herrschern über gesellschaftliche Klassen hin zu Künstlern. Das Zeichen bzw. Medium ,Geld‘ scheint also hinsichtlich seiner Gestaltung merkwürdig arbiträr zu sein. Das stimmt jedoch nur bedingt. Natürlich lassen sich verschiedenste Motive - Natur, Kunst, Berühmtheiten, Bauwerke, Tiere, Technik, Fabelwesen oder Götter - auf Banknoten drucken, aber eben nur in einer ganz bestimmen Darstellungsweise, d.h. in einer charakteristischen Bildsprache. Diese hat sich historisch ausgeformt und ist stark vom Produktionskontext beeinflusst. Letztlich zielt sie auf die Visualisierung des Abstraktums, Wert'. Sie benutzt dazu kein singuläres Motiv, sondern eben eine bestimmte Art der Darstellung. Hierbei werden die ursprünglichen Motive (etwa Gemälde oder Fotographien) dekontextualisiert, sie werden als Ausschnitt collageartig mit typischen Elementen wie formelhaften Währungssymbolen, Wertrahmen, Schmuckvignetten und Ornamenten kombiniert. Gleichzeitig werden sie - wie in einem Zerrspiegel durch jene charakteristische drucktechnisch bedingte Darstellungsweise (v.a. Guillochen) verändert. Diese maschinelle Ästhetik entstammt dem Versuch, in einem technisch-industriellen Prozess Vertrauen herzustellen, indem der für Banknoten erforderliche Herstellungsprozess möglichst aufwändig und komplex gemacht wird. Hinzu kommt allerdings der Faktor der Akzeptanz von Banknoten. Als Wertsymbol bedürfen sie einer allgemeinen Anerkennung, was ihre Gestaltung zu einer psychologisch äußerst sensiblen Angelegenheit macht. Aus diesem Grund werden häufig altbekannte Farbgebungen beibehalten, vertraute Referenzen hergestellt und obsolete Darstellungsweisen beibehalten. So entstand ein typischer Kanon an visuellen Gestaltungsmustern, die in unserer Wahrnehmung einen Papierstreifen zu einem Wertzeichen machen - egal, welche konkreten Motive darauf abgebildet sind. Indem sich nun eine visuelle Soziologie damit befasst, wie eine solche Bildsprache des Geldes (respektive des Wertes)

33 Im 19. Jahrhundert wurden bewusst halbnackte Frauen auf die Banknoten gedruckt. Man nahm an, dass die Scheine dadurch aufmerksamer betrachtet und so Fälschungen schneller entdeckt würden (Brion / Moreau 2001: 112). 
beschaffen ist, kann sie zu einem tieferen Verständnis des Funktionierens des symbolisch generalisierten Kommunikationsmediums Geld beitragen - konkret zur Tatsache, warum Menschen ein im Grunde nutzloses Symbol überhaupt annehmen. Die (systemtheoretische) Geldtheorie erklärt dies nämlich nur vage damit, dass anscheinend ,a certain institutionalized confidence in the monetary system" (Parson 1967: 307) besteht. Welcher Rolle dabei der konkreten Materialität - und damit auch Visualität - des Geldmediums zukommt, gilt es zu klären. Entsprechend könnte man danach fragen, welche gestalterischen Elemente eine Banknote zu einem Wertzeichen machen, so dass selbst noch nie gesehene Fremdwährungen auf Anhieb als ,Geld' erkannt werden? Welche Art(en) der Darstellung schaffen Vertrauen? Was ergeben Vergleiche mit anderen ,Wertdokumenten“ wie Aktien, Gutscheinen oder Vertragsdokumenten? Wie werden Geldscheine nach Vertrauensbrüchen (Währungskrisen) neu gestaltet?

Der zweite soziologisch relevante Aspekt, der sich hier eröffnet, bezieht sich auf die performativen Wirkungen von Banknoten. Wie werden Banknoten wahrgenommen, wie wirken sie auf ihre Nutzer? Ein Teil dieser Wirkung liegt sicherlich auf einer präreflexiven Ebene (man denke etwa an die These des , banal nationalism'). Analysieren lassen sich solche Wirkungen nur innerhalb der konkreten Geldpraxen. Am besten gelingt dies, wenn die epistemologische Ordnung aufbricht, d.h. wenn neues Material (Aktanten im Sinne der Akteur-Netzwerk-Theorie) in Praxen eingebaut wird (beispielsweise bei Währungsreformen). Für eine visuelle Soziologie am interessantesten ist hier sicherlich der Aspekt der Rezeption im Alltag. Wie werden Banknoten bzw. ihre gestalterischen Elemente wahrgenommen? Welche, auch abweichenden, ,Lesarten' ${ }^{34}$ entwickeln die Menschen den Scheinen gegenüber und welche ,eigensinnigen' Nutzungen resultieren daraus?

Der dritte Fragekomplex hat starke Überschneidungen mit der Kunstgeschichte, da hier Banknoten als Dokumente der Zeitgeschichte herangezogen werden. Dennoch finden sich hier auch genuin soziologische Fragestellungen, etwa indem anhand entsprechender Motive auf Banknoten Rückschlüsse auf den Wandel von Geschlechterrollen oder des Natur- und Technikverständnisses gezogen werden.

Insgesamt eröffnet sich hier ein weites empirisches Forschungsfeld, das neben den Bildern auf der Banknote auch die sozialen Praktiken ihrer Produktion, Interpretation und Verwendung berücksichtigen muss (Burri 2008: 345). Entsprechende methodologisch-methodisch ausgearbeitete Ansätze sind erst in den letzten Jahren entstanden (Breckner 2010: 9; Schnettler / Raab 2008). Auf Banknoten im Speziellen wurden sie noch nicht angewandt. Hier ist ein systematischer methodischer Zugang noch in weiter Ferne. Bislang finden sich eher ,freihändige' Interpretationen (etwa Helleiner 2003) neben quantitativen Inhaltsanalysen der abgebildeten Personen (etwa Hymans 2005); mal werden diskusanalytisch orientierte ikonographische Analysen durchgeführt oder methodenplurale Ansätze verfolgt (Interviews und Dokumentenanalysen; etwa Unwin / Hewitt 2001). Vielversprechender erscheinen einige Methoden der visuellen Soziologie, insbesondere die dokumentarische Methode (Bohnsack 2005, 2006, 2008) und die Segmentanalyse (Breckner 2008, 2010). Erstere ermöglicht nicht nur das textlich-narrative (Vor-)Wissen um den Bildinhalt methodisch zu verdrängen (Bohnsack 2006: 46) und so neue, noch nicht bedachte ,Botschaften“ von Banknoten herauszuarbeiten. Es erlaubt auch die Untersuchung der tatsächlichen Wirkung der Formalstruktur von Banknoten,

34 So wurde beispielsweise die 10,000-Mark-Reichsbanknote vom 19.1.1922 als „Vampirschein“ bezeichnet. Denn, drehte man das dort abgebildete Dürer-Porträt eines jungen Mannes um 90 Grad nach links, konnte man die Zeichnung eines vampirartigen Kopfes feststellen. In der schweren Zeit nach dem Ersten Weltkrieg interpretierte man den jungen Mann als Deutschland, der durch den Versailler Vertrag (Vampir) entkräftet wird (Pick 1992: 309). Ein anderes Beispiel sind Verschwörungstheorien, die sich um den Dollar ranken. In Spanien wird der 500-Euro-Schein als ,Bin Laden` bezeichnet, denn jeder hat schon von ihm gehört, aber niemand hat ihn je zu Gesicht bekommen. 
indem in einer „Kompositionsvariation“ (2006: 51) experimentell die Komposition verändert bzw. durch systematisch variiert wird (für ein ähnliches Verfahren siehe Heij 2012). Breckners vorwiegend für fotografische Bilder entwickelte Segmentanalyse wiederum lässt sich für die Untersuchung von Banknoten adaptieren. Dadurch können die unterschiedlichen collageartig zusammengesetzten Bildelemente von Geldscheinen (Porträts, Schmuckrahmen, Vignetten etc.) zunächst getrennt und dann in ihrem Zusammenspiel analysiert werden. Je nach Fragestellung und Perspektive müssen noch andere Verfahren herangezogen werden; angesichts der schieren Masse der Banknoten etwa die quantitativ ausgerichtete Inhaltsanalyse (Bell 2001; Rose 2012) oder für die Rezeption (quasi-)experimentelle Designs.

Man sieht, mit der Untersuchung von Banknoten tut sich ein hochinteressantes und vielfältiges Forschungsfeld für die visuelle Soziologie auf. ${ }^{35}$ Um den eingangs zitierten Aphorismus von Benjamin noch einmal aufzugreifen: Banknoten können uns sehr viel zeigen mehr als eine reine „Fassadenarchitektur der Hölle“.

\section{Literatur}

Ahonen, P. (1989): The Meaning of Money: Comparing a Peircean and Saussurean Perspective, in: R. Kevelson (Hrsg.), Law and semiotics. Volume 3, New York / NY, S. 13-30.

Anderson, B. (1993): Die Erfindung der Nation. Zur Karriere eines folgenreichen Konzepts, Frankfurt / Main - New York / NY.

Angus, I. (1975): Paper Money, New York / NY.

Athanassakis, I. (2008): Die Aktie als Bild. Zur Kulturgeschichte von Wertpapieren, Wien.

Barthes, R. (1990): Rhetorik des Bildes, in: Ders., Der entgegenkommende und der stumpfe Sinn, Frankfurt / Main, S. 28-46.

BBC News (7.11.2000): How to join the noteworthy, Beitrag von Ryan Dilley.

Bell, P. (2001): Content Analysis of Visual Images, in: T. van Leeuwen / C. Jewitt (Hrsg.), Handbook of Visual Analysis, London, S. 10-34.

Bender, K.W. (2004): Geldmacher. Das geheimste Gewerbe der Welt, Weinheim.

Benjamin, W. (1928): Einbahnstrasse, Berlin.

Billig, M. (1995): Banal Nationalism, London.

Blaazer, David (2002): Sterling Identities, in: History Today 52, S. 12.

Blumenberg, H. (1976): Geld oder Leben. Eine metaphorologische Studie zur Konsistenz der Philosophie Georg Simmels, in: H. Böhringer / K. Gründer (Hrsg.), Ästhetik und Soziologie um die Jahrhundertwende: Georg Simmel, Frankfurt / Main.

Bohnsack, R. (2005): Bildinterpretation und dokumentarische Methode, in: C. Wulf / J. Zirfas (Hrsg.), Ikonologie des Performativen, München, S. 246-262.

Bohnsack, R. (2006): Die dokumentarische Methode der Bildinterpretation in der Forschungspraxis, in: W. Marotzki / H. Niesyto (Hrsg.), Bildinterpretation und Bildverstehen, Wiesbaden, S. 45-75.

Bohnsack, R. (2008): The Interpretation of Pictures and the Documentary Method, in: FQS 9 / 3 Art. 26, abrufbar unter: http://www.qualitative-research.net/index.php/fqs/article/view/1171/2591, letztes Abrufdatum 20.3.2013.

Breckner, R. (2008): Bildwelten - Soziale Welten. Zur Interpretation von Bildern und Fotografien. OnlineBeitrag zu Workshop \& Workshow vom 23. / 24.11.2007.

Breckner, R. (2010): Sozialtheorie des Bildes. Zur interpretativen Analyse von Bildern und Fotografien, Bielefeld.

35 Hierzu ist 2014 eine interdisziplinäre Tagung geplant. Informationen beim Autor. 
Brion, R. / J.-L. Moreau (2001): A Flutter of Banknotes. From the First European Paper Money to the Euro, Antwerpen.

Burri, R.V. (2008): Bilder als soziale Praxis: Grundlegungen einer Soziologie des Visuellen, in: Zeitschrift für Soziologie 37, S. 342-358.

Christmann, G.B. (2008): The Power of Photographs of Buildings in the Dresden Urban Discourse. Towards a Visual Discourse Analysis, in: FQS 9 / 3, Art. 11, abrufbar unter: http://www.qualitativeresearch.net/index.php/fqs/article/view/1163, letztes Abrufdatum: 20.3.2013.

Cohen, B.J. (1998): The Geography of Money, Ithaca / NY.

Cox, F. / H.-W. Schmidt-Hannisa (Hrsg.) (2007): Money and culture, Frankfurt / Main.

Deutsche Bundesbank (1970): Frühzeit des Papiergeldes. Beispiele aus der Geldscheinsammlung der Deutschen Bundesbank, Frankfurt / Main.

Deutsche Bundesbank (2010): Das besondere Objekt. Ästhetik und Sicherheit - Weltbanknoten der Jahre 2005 bis 2009, abrufbar unter: http://www.bundesbank.de/download/bibliothek/publikationen/sonder_weltbanknoten.pdf, letztes Abrufdatum: 21.3.2012.

Deutschmann, C. (2000): Geld als , absolutes Mittel'. Zur Aktualität von Simmels Geldtheorie, in: Berliner Journal für Soziologie 10, S. 301-313.

Deutschmann, C. (2007): Wie harmlos ist Geld? Anmerkungen zur geldsoziologischen Diskussion, in: P. Kellermann (Hrsg.), Die Geldgesellschaft und ihr Glaube. Ein interdisziplinärer Polylog, Wiesbaden, S. 161-173.

Eco, U. (1994): Einführung in die Semiotik, München.

Europäische Zentralbank (EZB) (2011): Die Verwendung von Euro-Banknoten - Ergebnisse aus zwei Umfragen unter privaten Haushalten und Unternehmen. Monatsbericht April, S. 83-95.

Fuller, H. (2008): Civitatis Ghaniensis Conditor. Kwame Nkrumah, Symbolic Nationalism and the Iconography of Ghanaian Money 1957 the Golden Jubilee, in: Nations and Nationalism 14, S. 520-541.

Gabriel, G. (2002): Ästhetik und Rhetorik des Geldes, Bad Cannstatt / Stuttgart.

Gabriel, G. (2009): Die Ästhetik des Geldes, in: K.P. Liessmann (Hrsg.), Geld. Was die Welt im Innersten zusammenhält?, Wien, S. 264-289.

Galán, J.E. / M. Sarmiento (2008): Banknote Printing at Modern Central Banking: Trends, Costs, and Efficiency, in: Money Affairs 21, S. 217-262.

Ganßmann, H. (1996): Geld und Arbeit. Wirtschaftssoziologische Grundlagen einer Theorie der modernen Gesellschaft, Frankfurt / Main.

Gilbert, E. (1998): 'Ornamenting the Facade of Hell': Iconographies of 19th-century Canadian Paper Money, in: Environment and Planning D: Society and Space 16, S. 57-80.

Gilbert, E. / E. Helleiner (1999): Introduction - Nation-States and Money. Historical Contexts, Interdisciplinary perspectives, in: Dies. (Hrsg.), Nation-States and Money, S. 1-21.

Gilbert, E. / E. Helleiner (Hrsg.) (1999): Nation-States and Money. The past, present and future of national currencies, London.

Grabowski, H.L. / W. Haney (2008): Das Geld des Terrors. Geld und Geldersatz in deutschen Konzentrationslagern und Gettos 1933 bis 1945: Dokumentation und Katalog, Regenstauf.

Habermas, J. (1981): Theorie des kommunikativen Handelns. Band I und II, Frankfurt / Main.

Heij, H. de (2002): A Method for Measuring the Public's Appreciation and Knowledge of Banknotes, San Jose / CA.

Heij, H. de (2008): Banknote Opionion Poll: A Method for Collecting Costumer Feedback on Banknote Design, Amsterdem.

Heij, H. de (2010 a): Key Elements in Banknote Design. Part I: The Product Perspective, in: Keesing Journal of Documents \& Identity 32, S. 6-13. 
Heij, H. de (2010 b): Key Elements in Banknote design. Part II: The Process Perspective, in: Keesing Journal of Documents \& Identity 32, S. 14-18.

Heij, H. de (2012): Designing Banknote Identity.

Helleiner, E. (2003): The Making of National Money. Territorial Currencies in Historical Perspective, Ithaca.

Hewitt, V.H. / J.M. Keyworth (1987): As Good as Gold. 300 Years of British Bank Note Design, London.

Hewitt, V. (1994): Beauty and the Banknote. Images of Women on Paper Money, London.

Hewitt, V. (1995): The Banker's Art. Studies in Paper Money, London.

Hörisch, J. (1996): Kopf oder Zahl. Die Poesie des Geldes, Frankfurt / Main.

Huber, J. (2004): Reform der Geldschöpfung. Wiederherstellung des staatlichen Geldregals und der Seigniorage durch Vollgeld, in: Der Hallesche Graureiher 5, S. 1-31.

Hutter, M. (2007): Visual Credit:. The Britannia Vignette on the Notes of the Bank, in: F. Cox / H.-W. Schmidt-Hannisa (Hrsg.), Money and Culture, Frankfurt / Main, S. 15-36.

Hymans, J.E.C. (2004): The Changing Color of Money: European Currency Iconography and Collective Identity, in: European Journal of International Relations 10, S. 5-31.

Hymans, J.E.C. (2005): International Patterns in National Identity Content: The Case of Japanese Banknote Iconography, in: Journal of East Asian Studies 5, S. 315-346.

Hymans, J.E.C. (2006): Money for Mars? The Euro Banknotes and European Identity, abrufbar unter: http://www-bcf.usc.edu/ hymans/Hymansfishmess.pdf, letztes Abrufdatum: 11.7.2012.

Imdahl, M. (1995): Ikonik, in: G. Boehm (Hrsg.), Was ist ein Bild?, München, S. 300-324.

Kaelberer, M. (2004): The Euro and European Identity: Symbols, Power and the Politics of European Monetary Union, in: Review of International Studies 30 / 2, S. 161-178.

Kaelberer, M. (2005): Deutschmark Nationalism and Europeanized Identity: Exploring Identity Aspects of Germany's Adoption of the Euro, in: German Politics 14, S. 283-296.

Klose, D.O.A. (1999): Ade DM! Geschichte der Mark von 1945 bis zum Euro, München.

Klüßendorf, N. (2007): Money and Identity in a Divided Country, in: R. Cunz (Hrsg.), Money and Identity. Lectures about History, Design, and Museology of Money, Hannover, S. 29-54.

Knapp, G.F. (1924 / 1905): The State Theory of Money, London.

Komar, G. (1991): Geldzeichen, in: Zeitschrift für Semiotik 13, S. 345-365.

Kwon, H. (2007): The dollarization of Vietnamese ghost money, in: Journal of the Royal Anthropological Institute 13, S. 73-90.

Lambek, M. (2001): The Value of Coins in a Sakalava Polity: Money, Death, and Historicity in Mahajanga, Madagascar, in: Comparative Studies in Society and History 43, S. 735-762.

Löffler, B. (Hrsg.) (2010): Die kulturelle Seite der Währung. Europäische Währungskulturen, Geldwerterfahrungen und Notenbanksysteme im 20. Jahrhundert, München.

Luhmann, N. (1987 [1984]): Soziale Systeme, Frankfurt / Main.

Luhmann, N. (1988): Die Wirtschaft der Gesellschaft, Frankfurt / Main.

Luhmann, N. (1998[1987]): Die Gesellschaft der Gesellschaft, Frankfurt / Main.

Marx, K. (1961[1859]): Zur Kritik der Politischen Ökonomie. Marx-Engels-Werke (MEW 13), Berlin (DDR).

Marx, K. (1972[1847]): Elend der Philosophie. Marx-Engels-Werke (MEW 4), Berlin (DDR).

Meier, D. (2001): Tauschringe als besondere Bewertungssysteme in der Schattenwirtschaft: eine theoretische und empirische Analyse, Berlin. 
Michel, B. (2007): Fotografien und ihre Lesarten. Dokumentarische Interpretation von Bildrezeptionsprozessen, in: R. Bohnsack / I. Nentwig-Gesemann / A.-M. Nohl (Hrsg.), Die dokumentarische Methode und ihre Forschungspraxis, Wiesbaden, S. 93-123.

Miklautz, E. (2005): Die Produktwelt als symbolische Form, in: G.M. König (Hrsg.), Alltagsdinge. Erkundungen der materiellen Kultur, Tübingen, S. 43-61.

Mittermeir, R. (2006): Geld und Information, in: P. Kellermann (Hrsg.), Geld und Gesellschaft. Interdisziplinäre Perspektiven, Wiesbaden, S. 139-170.

Mwangi, W. (2002): The Lion, the Native and the Coffee Plant: Political Imagery and the Ambiguous Art of Currency Design in Colonial Kenya, in: Geopolitics 7, S. 31-62.

Nathorst-Böös, E. (1970): About Swedish Banknotes, in: Revue internationale d'histoire de la banque 3, S. 89-96.

Nöth, W. (2000): Handbuch der Semiotik, Stuttgart.

North, M. (1994): Das Geld und seine Geschichte. Vom Mittelalter bis zur Gegenwart, München.

Nöth, W. (2011): Visual Semiotics: Key Features and an Application to Picture Ads, in: E. Margolis / L. Pauwels (Hrsg.), The SAGE Handbook of Visual Research Methods, Los Angeles / CA, S. 298-316.

Ofonagoro, W.I. (1979): Trade and Imperialism in Southern Nigeria 1881-1929, New York / NY.

Panofsky, E. (2006): Ikonographie und Ikonologie. Bildinterpretation nach dem Dreistufenmodell, Köln.

Parsons, T. (1967): Sociological Theory and Modern Society, New York / NY.

Penrose, J. (2011): Designing the Nation. Banknotes, Banal Nationalism and Alternative Conceptions of the State, in: Political Geography 30, S. 429-440.

Pick, A. (1992): Papiergeldlexikon, Regenstauf.

Pointon, M. (1998): Money and nationalism, in: G. Cubitt (Hrsg.), Imagining Nations, Manchester, S. 227-245.

Priddat, B.P. (2003): „Geist der Ornamentik“. Ideogrammatik des Geldes: Allegorien bürgerlicher Zivilreligion auf Banknoten des 19. und 20. Jahrhunderts, in: D. Baecker (Hrsg.), Kapitalismus als Religion, Berlin, S. 19-34.

Raento, P. / A. Hämäläinen / H. Ikonen / N. Mikkonen (2004): Striking Stories: A Political Geography of Euro Coinage, in: Political Geography 23, S. 929-956.

Risse, T. (2003): The Euro between National and European Identity, in: Journal of European Public Policy 10, S. 487-505.

Ritzer, G. (1995): Expressing America - A Critique of the Global Credit Card Society, Thousand Oaks / CA.

Robertson, F. (2005): The Aesthetics of Authenticity: Printed Banknotes as Industrial Currency, in: Technology and Culture 46, S. 31-50.

Rose, G. (2012): Visual Methodologies. An Introduction to Researching with Visual Materials, Los Angeles / CA.

Rotman, B. (2000): Die Null und das Nichts. Eine Semiotik des Nullpunkts, Berlin.

Schnettler, B. / J. Raab (2008): Interpretative Visual Analysis. Developments, State of the Art and Pending Problems, in: FQS 9 / 3, Art. 31, abrufbar unter: http://www.qualitative-research.net/index.php/fqs/ article/view/1149/2555, letztes Abrufdatum: 20.3.2013.

Schramm, P. (1985): Der Mensch und sein Geld im Spiegel der Kunst, Taunusstein.

Sedillot, R. (1992): Muscheln, Münzen und Papier. Die Geschichte des Geldes, Frankfurt / Main.

Simmel, G. (1989[1900]): Philosophie des Geldes, Frankfurt / Main.

Spiegel Online (30.12.2011): D-Mark-Design. Der schönere Schein, Beitrag von Benjamin Maack, abrufbar unter: http://einestages.spiegel.de/static/topicalbumbackground/24142/der_schoenere_schein.html, letztes Abrufdatum: 25.3.2012. 
Spiegel Online (17.2.2012): Spaßgeld: Tscheche tauscht erfolgreich 1000-Eros-Schein, abrufbar unter: Abrufbar unter: http://www.spiegel.de/panorama/gesellschaft/tschechien-geldfaelscher-drucktnacktefrauen-auf-1000-euro-scheine-a-844274.html, letztes Abrufdatum: 7.9.2012.

Tappe, O. (2007): A New Banknote in the People's Republic - The Iconography of the Kip as a Representation of Ideological Transformations in Laos (1957-2006), in: Internationales Asienforum 38, S. 87-108.

Tschoegl, A.E. (2004): Change the Regime - Change the Money: Bulgarian Banknotes, 1885 to 2003, in: Balkanologie 8, S. 7-31.

Thiel, C. (2012): Das „bessere“ Geld: Eine ethnographische Studie über Regionalwährungen, Wiesbaden.

Uemura, T. (2007): Japanese Paper Notes and German Printing Technology at the Beginning of the Meji Era (1868-1883), in: R. Cunz (Hrsg.), Money and Identity. Lectures about History, Design, and Museology of Money, Hannover, S. 129-139.

Unwin, T. / V. Hewitt (2001): Banknotes and National Identity in Central and Eastern Europe, in: Political Geography 20, S. 1005-1028.

Veselkova, M. / J. Horvath (2011): National Identity and Money: Czech and Slovak Lands 1918-2008, in: Nationalities Papers 39, S. 237-255.

Vilar, P. (1984): Gold und Geld in der Geschichte. Vom Ausgang des Mittelalters bis zur Gegenwart, München.

Walburg, R. (2011): „,..für alle Fälle...“ - Die geheimnisvollen Banknoten aus der Zeit der Deutschen Mark, in: Deutsche Bundesbank (Hrsg.), Geldgeschichte im Geldmuseum 2010, Frankfurt / Main, S. 61-106.

Waldenfels, B. (1995): Ordnungen des Sichtbaren, in: G. Boehm (Hrsg.), Was ist ein Bild?, München, S. 233-252.

Wallach, Y. (2011): Creating a Country through Currency and Stamps: State Symbols and Nation-Building in British-Ruled Palestine, in: Nations and Nationalism 17, S. 129-147.

Warburg, A. (1998): Die Erneuerung der heidnischen Antike. Kulturwissenschaftliche Beiträge zur Geschichte der europäischen Renaissance, Berlin.

Weatherford, J. (1999): Eine kurze Geschichte des Geldes und der Währungen, Oesch.

Weber, M. (1972[1921]): Wirtschaft und Gesellschaft. Grundriss der verstehenden Ökonomie, Tübingen.

Weimer, W. (1994): Geschichte des Geldes. Eine Chronik mit Texten und Bildern, Frankfurt / Main.

Winkler, H. (2004): Diskursökonomie. Versuch über die innere Ökonomie der Medien, Frankfurt / Main.

Winter, H. (2007): The Design of the Euro Banknotes, in: R. Cunz (Hrsg.), Money and Identity. Lectures about History, Design, and Museology of Money, Hannover, S. 81-101.

Zelizer, V. (1994): The Social Meaning of Money, New York / NY.

Dr. Christian Thiel

Universität der Bundeswehr München Institut für Soziologie und Volkswirtschaftslehre

Geb. 033, R. 3123

Werner-Heisenberg-Weg 39

D-85577 Neubiberg

Christian.Thiel@unibw.de 